\title{
برامج التعاون اللامركزي وغير الحكومي الأوروبي - العرببي بوصفها آلية استجابة للهجرة القائهرية القسرية: "برنامج اللاجئين والمجتمعات المتوستوسطية المضيفة" نموذجًا
} European-Arab Decentralized and Non-Governmental
Cooperation Programs as a Forced Migration Response
Mechanism: A Case Study of "Mediterranean Refugees
and Host Communities Program"

تختبر هذه الدراسة قدرة برامج التعاون اللامركزي الأوروبي - العربهي على الاستجابة لأزمة اللجوء الحالية وتأطير

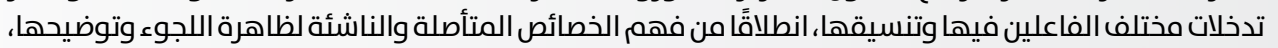

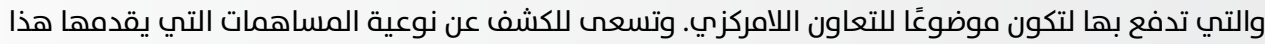

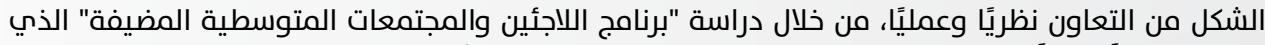

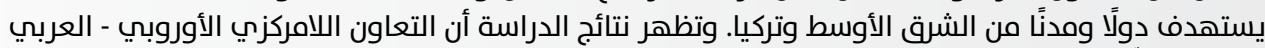

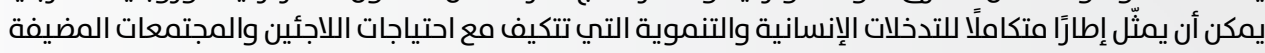

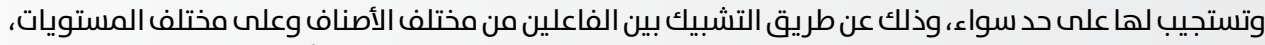

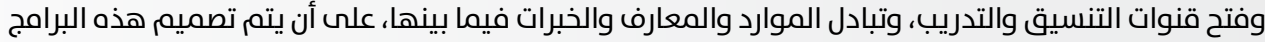

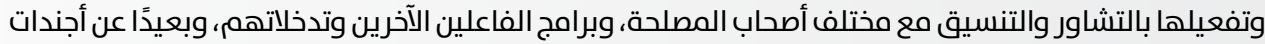
سياسية واستراتيجية تُصنع وتُنفذ على حساب اللاجئين والدول والمدن المضيفة.

كلمات مفتاحية: الهجرة القسرية، اللجوء، التعاون اللامركزي، مدن اللجوء، سياسات الهجرة في أوروبا، اللاجئون العرب.

This study examines the ability of European-Arab decentralized cooperation programs to respond to the current refugee crisis and to coordinate the interventions of various actors. The study seeks to understand the inherent and emerging characteristics of the refugee phenomenon that make it a subject of decentralized cooperation before turning to look at the different theoretical and practical contributions of this form of cooperation. It examines Refugee and Mediterranean Host Cities Program, which targets countries and cities in the Middle East and Turkey. The results of the study show that decentralized Euro-Arab cooperation can constitute an integrated framework for humanitarian and development interventions that adapt and respond to the needs of both refugees and host communities. This can be achieved by networking between various actors; opening channels of coordination and training; and exchanging resources, knowledge and experience. These programs should be designed and implemented in consultation with various stakeholders and in coordination with other programs and interventions, far removed from political and strategic agendas that exist at the expense of refugees, host countries and cities.

Keywords: Forced Migration, Asylum, Decentralized Cooperation, Host Cities, European Migration Policies, Arab Refugees. 
مع تحول تدفقات اللاجئين مؤخرًا من المخيمات إلى المدن والتجمعات السكنية، تزايدت الطبيعة الحضرية لظاهرة الهجرة القسرية (النزوح واللجوء)، وخاصة في المنطقة العربية، وأصبحت هجرةً طويلة الأمد. وبذلك أضيفت إشكاليات وتحديات جديدة إلى الإثكاليات التي تثيرها نُهج التعامل التقليدية القائمة على مساعدة النازحين

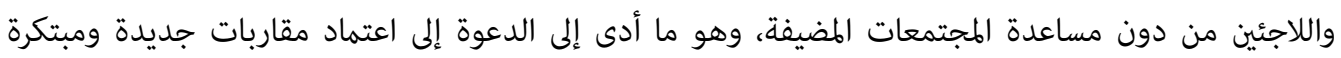
للتعامل مع الظاهرة، ومقاربات ترتكز أساسًا على التعاون والتنسيق الوثيق مع السلطات (الجماعات) المحلية. وهنا يبرز "التعاون اللامركزي"(1) بمقارباته المتعددة المستويات والفواعل، بوصفه أحد أشكال التعاون التي يُراهَن عليها لمساعدة المدن والمجتمعات المضيفة للاجئين (من الشمال والجنوب) على تسيير هذه الظاهرة/ الأزمة والتحديات

المتعددة الأبعاد المصاحبة لها.

في الوقت الذي تعرف فيه منطقة المتوسط، بضفتيه الأوروبية والعربية، واحدة من أكبر أزمات الهجرة القسرية وأشدها منذ الحرب العالمية الثانية، ازداد تململ الدول والمدن والمجتمعات المحلية المضيفة. وبرزت مطالب المسؤولين المحليين بضرورة تنسيق جهود الفاعلين والمتدخلين (خاصة المنظمات غير الحكومية) مع السلطات (الجماعات) المحلية. وتجاوبًا مع ذلك، تمّ إطلاق عدة برامج للتعاون اللامركزي في مجال هجرة اللجوء، يشمل العديد منها الدول

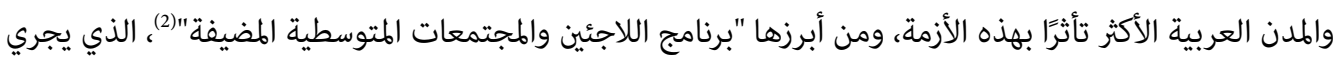
تنفيذه حاليًا، في سياق جدل كبير حول سياسة اللجوء في المنطقة والعالم، وجدّية الأطراف المعنية في تقديم الحلول المناسبة والفعالة عبر هذا الشكل من التعاون وغيره.

ومع حدوث تحول مهم في نموذج التعامل مع ظاهرة اللجوء الذي تكرّس مع الاتفاق العالمي بشأن اللاجئين، الذي يقر مسؤولية الحكومات والفاعلين المحليين ودورهم في هذا المجال، ويدعو إلى تكثيف التنسيق والتعاون اللامركزي فيما بينهم، تبرز الحاجة إلى تقديم إجابة عن سؤال: ما مدى أهلية التعاون اللامركزي الأوروبي - العربي وفاعليته

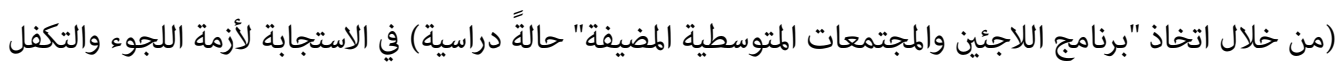
بانشغالات وتحديات اللاجئين والمدن والمجتمعات العربية المضيفة لهم؟ ويتفرع عن هذا السؤال عدة أسئلة، هي: ما مسوغات التحول نحو التعامل اللامركزي المتعدد الفواعل والمستويات مع هجرة اللجوء؟ فيمَ تتمثل المساهمات

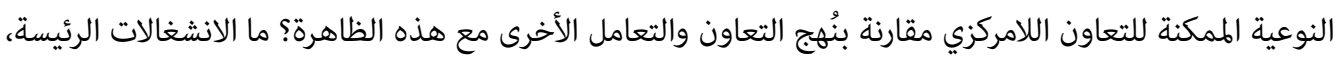

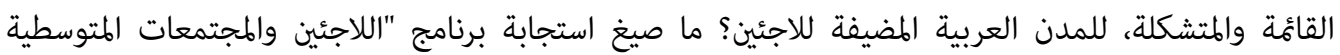
المضيفة" ودرجاتها، من الناحية النوعية على الأقل، لانشغالات اللاجئين والمدن العربية المشمولة به؟

1

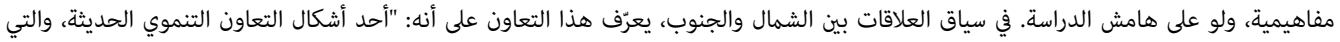

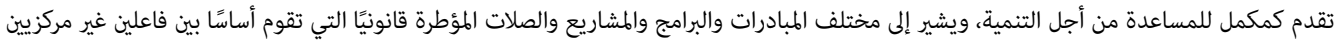

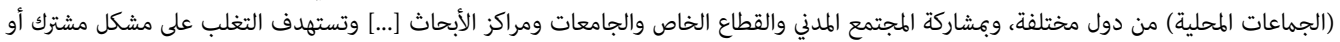

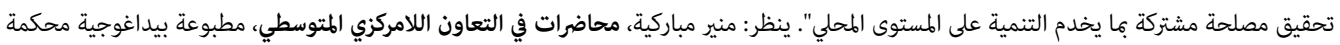

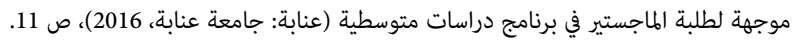

2 "Refugees and Host Communities," Center for Mediterranean Integration, accessed on 28/1/2020, at: http://bit.ly/36laBi3 
وانطلاقًا من مسح أحدث الأدبيات ومراجعتها حول التعاون اللامركزي الأورومتوسطي في مجال الهجرة بأشكالها المختلفة،

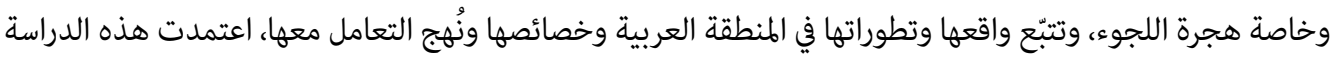
على فرضيتين أساسيتين لتوجيه عملية البحث؛ الأولى هي خصائص الهجرة القسرية العربية (النزوح واللجوء)، خاصة

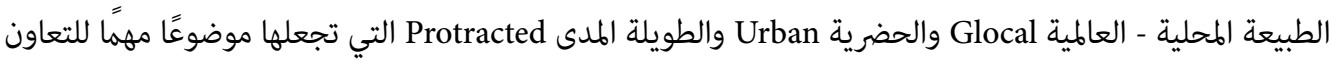
اللامركزي الأوروبي - العربي (المتوسطي بشكل عام). أما الفرضية الثانية فهي: رغم المساهمات النوعية "المتكاملة"

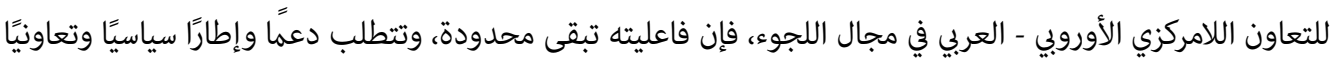

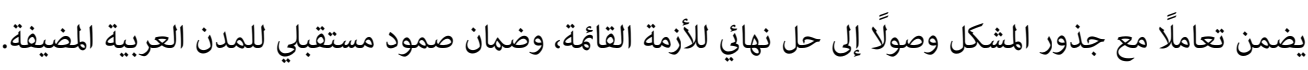
تتجلى أهمية هذه الدراسة في انشغالها، على نحو بارز، بأشكال التعاون التنموي الدولي الذي يحظى باعتراف دولي متزايد، نظرًا إلى قدرته على تنسيق وحكامة جهود الفاعلين والمتدخلين في مجالات عديدة، من بينها الهجرة الدولية بمختلف أشكالها. وتزداد أهمية الخوض في هذا التعاون في حالة هجرة اللجوء والنزوح التي تشتكي الأطراف المعنية (وخاصة اللاجئين وسلطات المدن والبلديات المضيفة) من عدم ملاءمة التدخلات، وتفرّق جهود المتدخلين وتشتتها،

$$
\text { وضعف التنسيق مع الحكومات والسلطات المحلية. }
$$

وتزداد أهمية الدراسة وضوحًا بإبراز أهم أهدافها، والمتمثلة أولًا، في التعرف إلى نوعية الاستجابة والحلول المبتكرة التي تقدمها برامج التعاون اللامركزي لانشغالات المدن والمجتمعات المحلية العربية المعنية بالهجرة القسرية (هجرة اللجوء خصوصًا). ثانيًا، تحديد مدى فاعلية الاستجابات والحلول التي يقدمها هذا الشكل من التعاون، مقارنة بنُهج

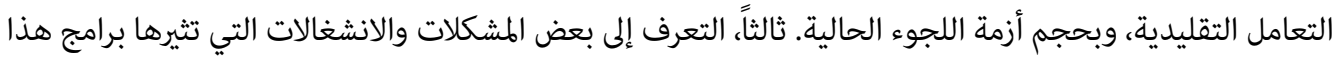

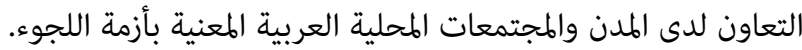

ومن ناحية منهج التناول والإطار التحليلي العام، تختبر هذه الدراسة فاعلية برامج التعاون اللامركزي الإقليمي

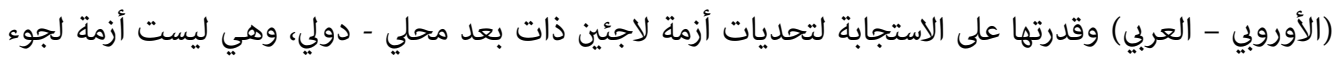

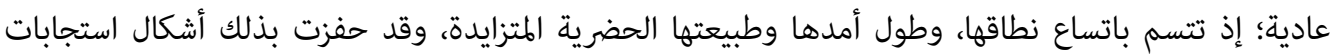
متعددة ومختلفة، وبعضها مبتكر، من طرف فاعلين متعددين، كما تعددت أيضًا برامج ومشاريع التعاون اللامركزي

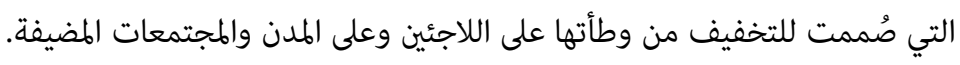

ومن أجل التمكن من اختبار أكبر قدر ممكن من أشكال الاستجابات والمساهمات التي يكنن أن يقدمها التعاون

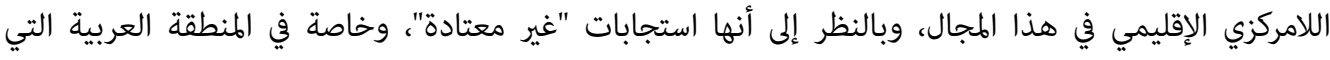
تضعف فيها اللامركزية، وعلاقات التعاون اللامركزي، والحوكمة المتعددة المستويات لقضايا الهجرة، وتغلب فيها مقاربات التعامل التقليدية (الإنسانية) التي تقودها المنظمات الدولية الحكومية وغير الحكومية، وجب علينا

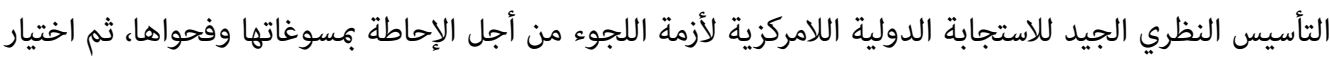
برنامج تعاون لامركزي إقليمي يتسم بشمولية الاستجابة وتكامل عناصرها، وتحليله وتقييمه في ضوء المساهمات النظرية المتوقعة منه، واحتياجات اللاجئين والمدن المضيفة المعبّر عنها في تقارير مسوح ودراسات استطلاعية حديثة أجرتها مؤسسات متخصصة في هذا المجال، وأيضًا الاستطلاعات التي أجراها البرنامج ذاته على مستوى الدول والمجتمعات المضيفة المستهدفة. 
وتجدر الإشارة إلى أن تحليل برامج التعاون اللامركزي وتقيمها يخضعان لخطوات وإجراءات منهجية خاصة، تراعي خصوصية هذا الشكل من التعاون ونوعية مساهماته؛ فتنطلق من تحليل "هندسة البرنامج" ونقدها، رجوعًا إلى بلى وثائقه الرسمية، ووقوفًا عند سياق بعثه، والأطراف المشرفة والداعمة والشريكة، وأهدافه وتدابيره العملية (تتضمن

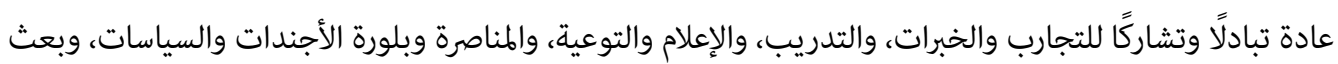

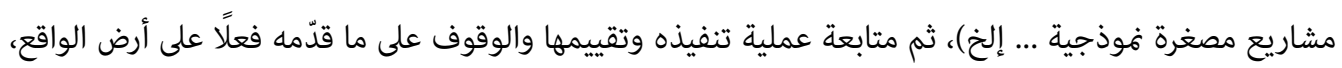
وانتهاء بتقييم آثاره الممتدة والطستدامة (الدروس المستفادة، والمبادئ الموجهة، وأطر الحوكمة وغيرها).

تتوزع هذه الدراسة على أربعة محاور: يُبرز المحور الأول الخصائص الأصيلة والناشئة لهجرة اللجوء التي وجب

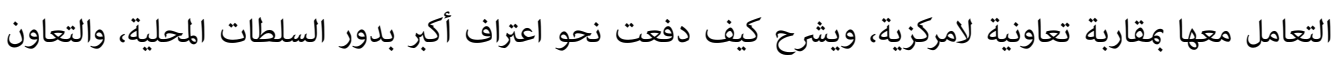

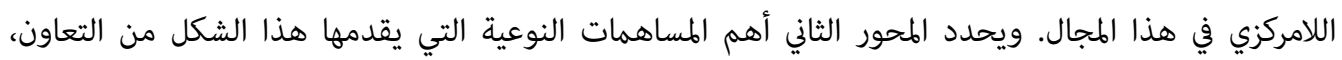
عندما يأتي على شكل برامج متكاملة. ويغطي المحوران الأخيران الجانب التطبيقي؛ فيعرض الثالث أهم احتياجات

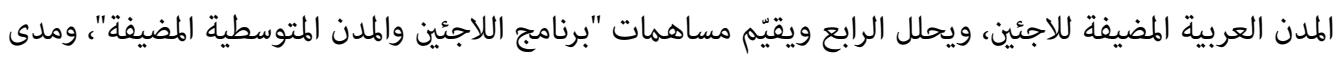
استجابته لتلك الاحتياجات من خلال تأطير وتوجيه التعاون مع المدن الأوروبية والعربية المشمولة بالبرنامج، وبينه مسينها.

\section{أولًا: هجرة اللجوء بوصفها موضوعًا للتعاون اللامركزمي}

ظلت هجرة اللجوء، بطبيعتها وحجم رهاناتها، موضوعًا للتعاون الدولي الحكومي وغير الحكومي (ما بين المنظمات غير الحكومية) الإنساني الذي يأتي تحت مظلات ومسميات مختلفة. لكن تحولات عديدة على مختلف الصعد في العقود الأخيرة،

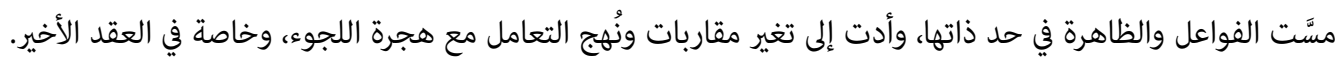
مسَّ هذا التحول، أساسًا، طبيعة التعامل والتعاون المطلوبين، ليتحول من الطابع الإنساني والإغاثي الصرف، إلى

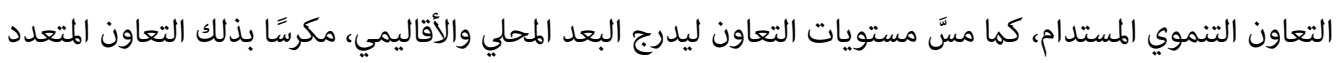

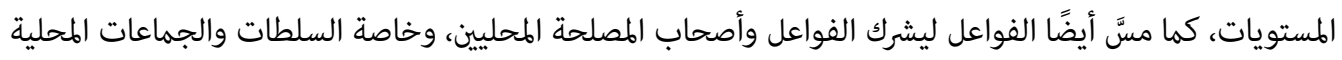
على مستوى الأقاليم والمدن. إن جوهر هذا التحوّل، المترافق مع تقدّم مسارات اللامركزية والاعتراف التدريجي

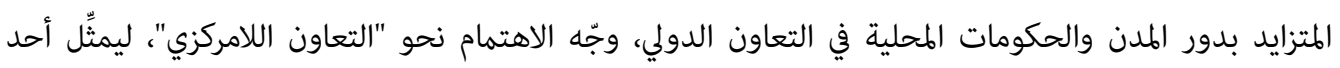

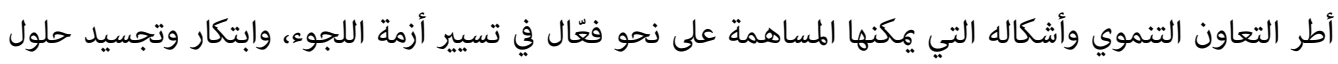
تستجيب لحاجات اللاجئين والمجتمعات المضيفة على المديين المتوسط والبعيد.

نحاول في هذا السياق تحديد وفهم خصائص هجرة اللجوء التي تجعل منها موضوعًا للتعاون اللامركزي، بل تجعل

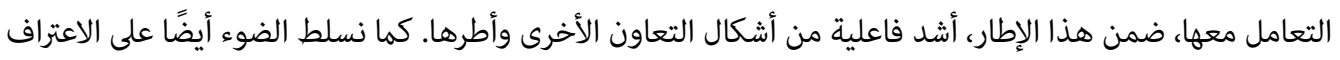
الدولي المتزايد بدور هذا الشكل من التعاون وأهميته.

\section{1. خصائص هجرة اللجوء وموجبات التعامل اللامركزي معها}

تشير قوانين غالبية الدول، في مقارباتها لعلاقات التعاون اللامركزي في جانبها المتعلق بهجالات التعاون، إلى أن التعاون يسري على القضايا والمواضيع التي تدخل ضمن مهمات السلطات المحلية وصلاحياتها. ولكنه عمليًا، يسري 
على القضايا التي غالبًا ما تكون لها مجموعة خصائص تحتم التعامل معها والتعاون عليها بهذا الشكل. وفيما يلي استعراض وإسقاط لأهم تلك الخصائص على هجرة اللجوء.

\section{أ. الطبيعة المحلية - العالمية}

الهجرة الدولية، بمختلف أشكالها، هي مسار "محلي - دولي - محلي"؛ فالمهاجرون إراديًا أو قسريًا ينطلقون من مدينة أو إقليم معيّن في دولة ما، متجهين نحو مدينة أو إقليم في دولة أخرى. وعلى المستوى المحلي، يقيم المهاجرون

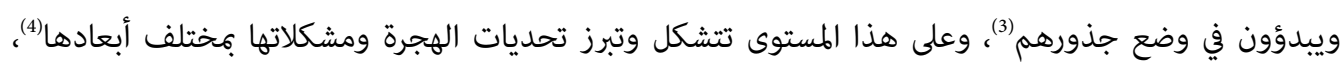
ومنه تنتشر وطنيًا وإقليميًا ودوليًا.

وعلى هذا الأساس، يعتبر الفضاء المحلي فضاءً مناسبًا للتسيير الفعال لواقع الهجرات، وذلك على الرغم من خضوعها للسلطة التنظيمية العامة للدول(5). ففي أغلب الدول، لا تمتلك سلطات المدن والبلديات صلاحية قانونية صريحة للتعامل مع طالبي اللجوء واللاجئين، ومع ذلك، فإن حجم القدوم وردّات الفعل البطيئة من السلطات الوطنية جعلا سلطات المدن في الواجهة، ودفعاها إلى أداء دور من دون صلاحية أو تفويض قانوني، ولا أيّ ميزانية لتغطية ذلك، فالمدن والحكومات المحلية هي التي توفر الحاجات الأساسية للمهاجرين واللاجئين القادمين الجدد، وهي التي تعمل على إدماج المهاجرين واللاجئين القدامى(6). لذا، هناك اعتراف تدريجي بالأهمية الحيوية للحكومات المحلية في مجال تسيير عمليات الهجرة وتدفقاتها(7)، رغم تجاهلها عادة بالنظر إلى غياب الصلاحيات القانونية - السياسية

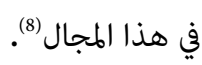

لا يعني القول بالطابع المحلي لهجرة اللجوء أن السلطات والفاعلين المحليين قادرون وحدهم، أو يمتلكون الصلاحية الواقعية الحصرية للتعامل معها، وإفا يتطلب ذلك تدخل عديد الفاعلين؛ فاستجابة البلديات لا يمكن تحسينها من دون جهد مشترك من المنظمات والمانحين والحكومات الوطنية للعمل معًا على تلبية حاجات المجتمعات المضيفة واللاجئين (9. وهذا المطلوب لا يمكن تحقيقه من دون اللجوء إلى التعاون اللامركزي. وفي ضوء ذلك، أصبحت الهجرات جزءًا من المحتوى المتعلق بعلاقات التعاون اللامركزي (10).

3 Mariel Araya \& Xavier Godàs, Migrations: A New Era of Co-peration between Local Governments (Montevideo: Observatorio de Cooperación Descentralizada UE-AL, 2008), p. 39.

4 Albert Serra \& Mireia Belil, "Local Governments and Migrations in Latin America and the European Union," in: Yearbook for Decentralised Cooperation: 2007 (Montevideo: Observatorio de Cooperación Descentralizada UE-AL, 2008), pp. 113, 114.

5 Araya \& Godàs, p. 43.

6 "Social Affairs: Refugee Reception and Integration in Cities," Eurocities (March 2016), p. 13, accessed on 5/2/2021, at: https://bit.ly/36PVk9m; "Urban Migration: Strengthening Cooperation with Civil Society," Peer Learning Note, no. 25, UCLG Learning (2019), p. 6, accessed on 5/2/2021, at: http://bit.ly/2MASWMP

7 Araya \& Godàs, p. 42.

8 Ibid., p. 40.

9 "Engaging Municipalities in the Response to the Syrian Refugee Crisis in Lebanon," Policy Brief, Mercy Corps (March 2014), p. 16, accessed on 5/2/2021, at: https://bit.ly/3rwGnkf

10 Araya \& Godàs, p. 40. 


\section{ب. السياقية الإقليمية أو المناطقية}

تختلف خبرات وتجارب الهجرة القسرية إلى المدن - بالنسبة إلى القادمين والمضيفين - اختلافًا كبيرًا، وفي بعض الأحيان

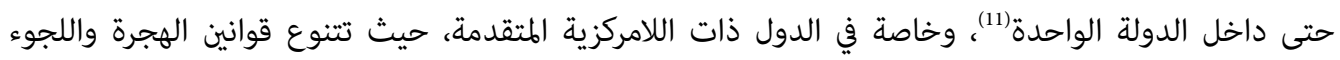

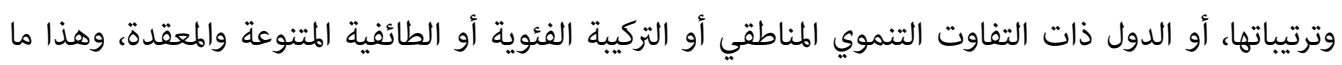
يجعل الاستجابات الوطنية التي تتخذ شكل سياسة عامة وطنية موحدة ومفروضة من الأعلى نحو الأسفل غير مجدية

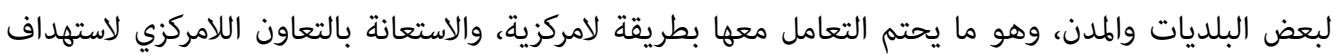
المناطق بما يناسبها، وتكملة التعاون الحكومي وغير الحكومي على مستواها.

\section{ج. الطبيعة الجوارية}

تجعل الخصائص السالفة الذكر من الهجرة ظاهرة "جوارية" بطبيعتها، إذ تؤثر هجرات اللجوء مباشرة في المجتمعات

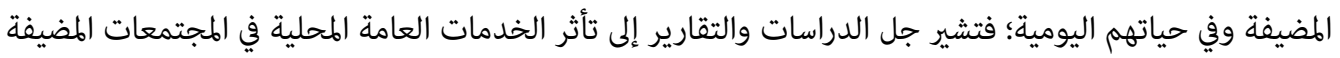

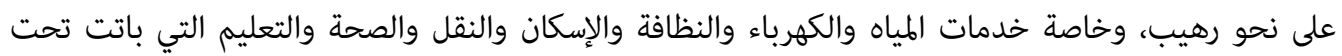

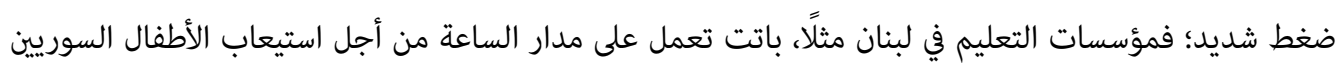
اللاجئين، كما تأثرت أسواق العمل تأثرًا كبيرًا أيضًا.

كما تساهم تحولات وعوامل أخرى عديدة في زيادة وتكريس الطبيعة "الجوارية" لهجرة اللجوء وأساليب التعامل

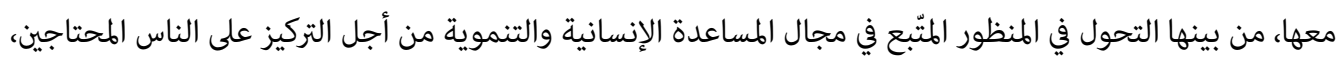

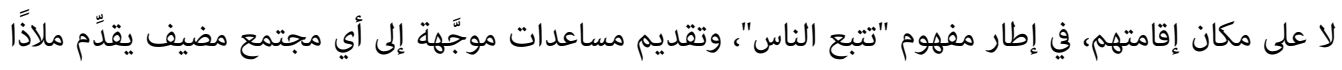

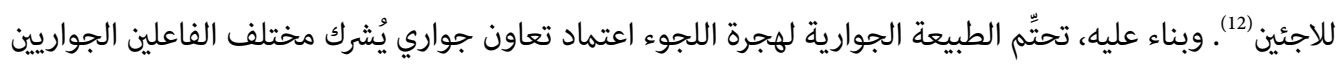

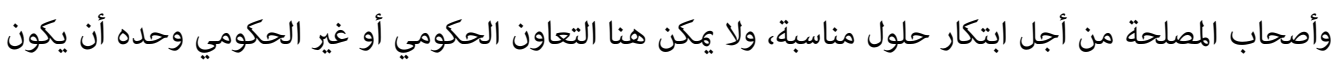
فعالًا في مثل هذه الحالات.

\section{د. الطبيعة الفنية}

لهجرة اللجوء بعدٌ فنّي أيضًا، مع تأكيد طابعها المتعدد الأبعاد، خاصة البعدين السياسي والاقتصادي - الاجتماعي،

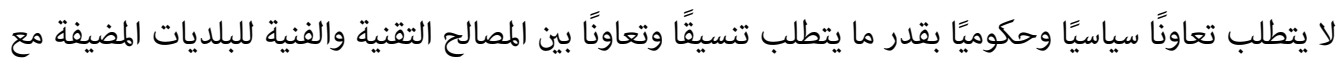

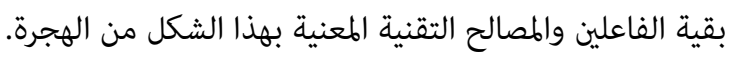

ويتجلى البعد الفني أساسًا في تسجيل اللاجئين وإحصائهم، وتسوية وضعياتهم المدنية، والتعرف إلى خلفياتهم

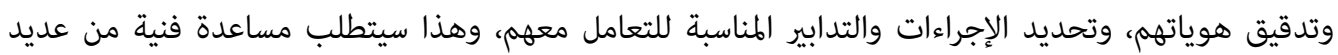

11 Robert Muggah \& Adriana Erthal Abdenur, "Refugees and the City: The Twenty-first-century Front Line," World Refugee Council Research Paper, no. 2, World Refugee Council \& Center for International Governance Innovation (July 2018), p. 11, accessed on 5/2/2021, at: https://bit.ly/3p31oRU

12 "اليوم العالمي للاجئين 2019: إعادة كتابة قواعد مساندة اللاجئين والمجتمعات امضيفة في الشرق الأوسط وشمال أفريقيا"، البنك الدولي، 2019/6/21/21اك،

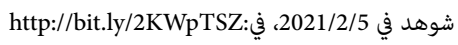


الفاعلين المختصين وأصحاب الخبرة والتجربة، وخاصة المنظمات الدولية وغير الحكومية ومصالح البلديات من الدول الأوروبية والغربية ذات السبق والتجربة.

\section{هـ. الطبيعة الحضرية}

مع بداية القرن الحالي، كانت غالبية اللاجئين تقيم في المخيمات أو التجمعات الريفية. ولكن منذ عام 2006، ارتفعت نسبة اللاجئين الحضريين على مستوى العالم ارتفاعًا كبيرًا، حتى وصلت إلى 61 في المئة عام 2018. وكان تدفق اللاجئين السوريين منذ عام 2012 قد تسبب في زيادة اللاجئين الحضريين إلى أكثر من الضعفئي (13).

وتتضح الطبيعة الحضرية لهجرة اللجوء جليًا في منطقة الشرق الأوسط وشمال أفريقيا عالية التعمي؛ إذ إن ما يقدر بنحو 80-90 في المئة من المهجرين يعيشون في المدن (14). وتصل نسبتهم في منطقة البحر المتوسط إلى 90 في المئة، وهذا مرتفع على نحو واضح عن المتوسط العالمي المقدر بنحو 61 في المئة (15).

على هذا النحو، أصبحت الهجرة تقريبًا مرادفًا للتحضر (الإقامة في المناطق الحضرية)، ويعزى ذلك إلى كون المدن هي

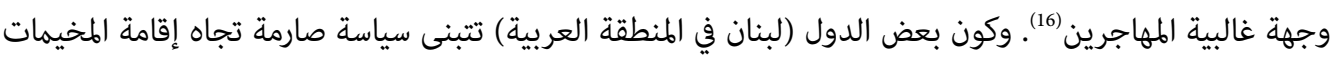

(سياسة "لا مخيم"، no-camp' policy')، لذلك تغلب الطبيعة الحضرية على نحو صارخ على أزمة اللجوء فيها.

تحتّم الطبيعة الحضرية لهجرة اللجوء على السلطات المحلية وقادة المدن التصرف والتعامل مع هذا الواقع، كما أنها تضفي طبيعة مخفيّة على هذه الهجرة، وتنتج عديد التحديات والصعوبات، مثل صعوبة تعقب اللاجئين والتعرف إلى عددهم وأماكن وجودهم، وفي الحصيلة، قد يحرمون حتى من المساعدات الإنسانية. وهذا ما يتطلب اللجوء إلى هنى

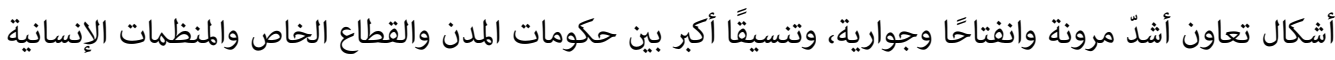
من أجل الاستفادة من مواطن القوة النسبية لكل هيئة من الهيئات المذكورة (17). وبناء عليه، فإن واقع النزوح الحضري يتطلب مقاربات مختلفة للاستجابات الإنسانية في الأوساط الحضرية؛ مقاربات تضع سلطات المدن في المركز، ليس على أساس القدرات، ولكن على أساس الرغبة والإرادة لاستضافة السكان النازحين، وشرعيتها للإشراف على تقديم الخدمات ضمن نطاق صلاحياتها 18.) وقد وجه تقرير الهجرة العالمي لسنة 2015

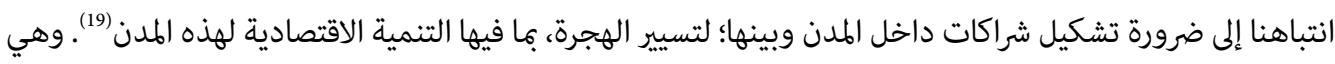

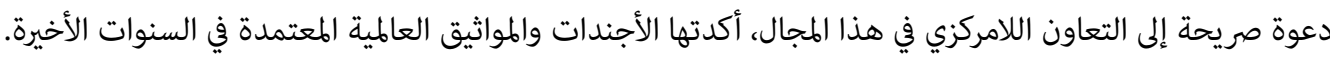

13 United Nations High Commissioner for Refugees, Global Trends: Forced Displacement in 2018 (Geneva: UNHCR, 2019), p. 57.

14 "Cities of Refuge in the Middle East: Bringing an Urban Lens to the Forced Displacement Challenge," Policy Note, The World Bank, 14/9/2017, p. 4 , accessed on 5/2/2021, at: https://bit.ly/3jtekiU

15 Ibid.

16 International Organization for Migration, World Migration Report 2018 (Geneva: IOM, 2017), p. 227.

17 "Urban Refuge: How Cities Are Building Inclusive Communities," International Rescue Committee (2018), p. 11, accessed on 5/2/2021, at: https://bit.ly/3oTcYPC

18 Ibid.

19 World Migration Report 2018, p. 229. 
ما برح متوسط مكوث اللاجئين في بلد اللجوء يزداد، حتى أصبحت هذه الهجرة هجرةً قسرية ممتدة (طويلة الأمد).

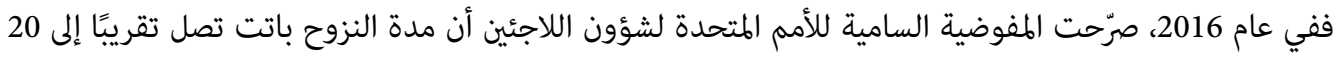
عامًا. وفي تقريرها الأخير، راجعت المفوضية تعريف حالات الهجرة القسرية الممتدة (20). وبناء عليه، فإن 15.9 مليون لاجئ هم في وضعيات ممتدة في نهاية عام 2018، وهذا يمثل 78 في المئة من اللاجئين حول العالم، مقارنةً بـ 66 في

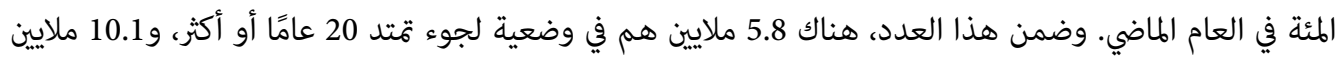

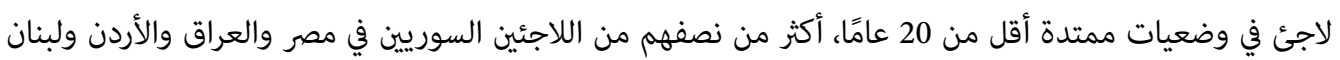

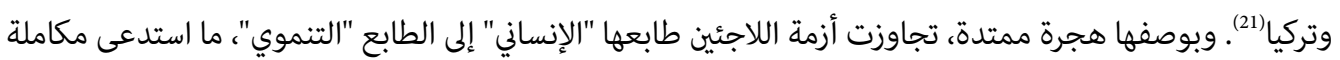
التعاون والتدخل الإنساني بالتعاون التنموي اللامركزي.

\section{2. تزايد الاعتراف بأهمية التعاون اللامركزي في مجال هجرة اللجوء}

تقر اتفاقية عام 1951 الخاصة بأوضاع اللاجئين بأنَّ التوصل إلى حل مُرضٍ لحالات اللاجئين لن يتحقق بمعزل عن التعاون

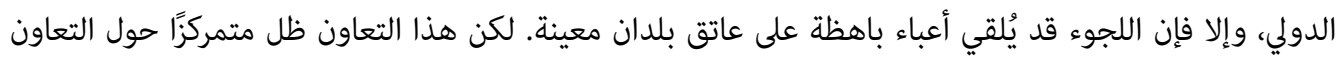

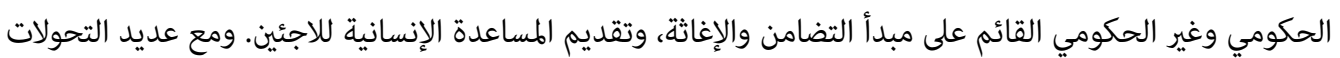
التي أشرنا إليها في مقدمة هذا المحور، وبالنظر إلى الخصائص المذكورة التي اتخذتها هذه الظاهرة، بدأ الاعتراف تدريجيًا بصلة السلطات المحلية والهيئات اللامركزية ومكانتها ضمن علاقات التعاون الدولي في مجال هجرة اللجوء.

وقد اعترفت العديد من المنظمات الدولية الفاعلة (خاصة الأمم المتحدة ووكالاتها، والاتحاد الأوروبي)، وكذلك الأطر

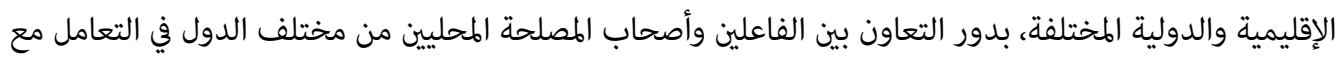
أزمة اللجوء من خلال التعاون اللامركزي. ولضيق المجال، ستقتصر الدراسة على الإشارة إلى التطورات الأخيرة في هذا

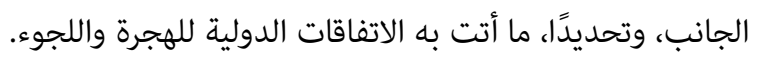

\section{أ. الاتفاق العالمي حول الهجرة الآمنة والنظامية والمنتظمة}

يعترف "الاتفاق العالمي حول الهجرة الآمنة والنظامية والمنتظمة" The Global Compact for Safe, Orderly and Regular Migration, GCM

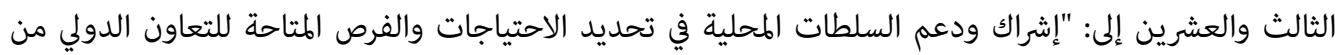

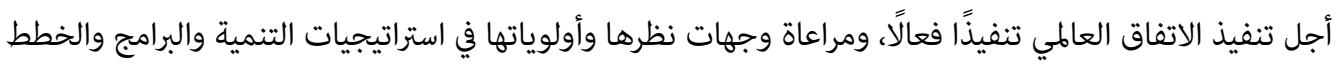

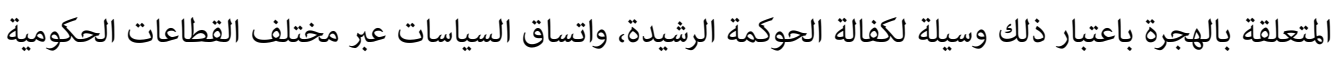
والسياساتية، ولإضفاء أقصى حد من الفعالية على التعاون الدولي في مجال التنمية وتحقيق أقصى أثر من هذا التعاون"(22). 
كما يعترف الميثاق بأن تنفيذه يتطلب جهودًا على مختلف المستويات بما فيها المستوى المحلي(23)، وأنه سيتم مع

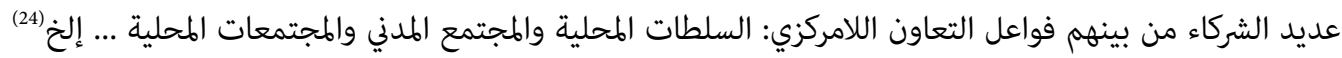
واعترف كذلك بدور الجماعات المحلية في التقييم والمتابعة والاستعراض الخاصة بتنفيذ الميثاق(25).

\section{ب. الاتفاق العالمصي بشأن اللاجئين}

يشير "الاتفاق العالمي بشأن اللاجئين The Global Compact on Refugees, GCR إلى أننا أمام حالة من "حالات اللجوء الكبرى" التي تحتاج إلى تعامل شامل ومتكامل، ومن الطبيعي أن تلتمس الدول والمجتمعات المضيفة الدعم من المجتمع الدولي. وقد أقر هذا الاتفاق تجاوز نُهج التعامل التقليدية مع حالات اللاجئين واسعة النطاق(26). وأقر أيضًا بأن "السلطات المحلية والفاعلين المحليين الآخرين، هم المستجيب الأول لحالات اللجوء واسعة النطاق، وهم الأكثر عرضة للضغوط الكبيرة على الملى المتوسط"(27). ويؤكد مسؤولية الجماعات المحلية ودورها في هذا المجال،

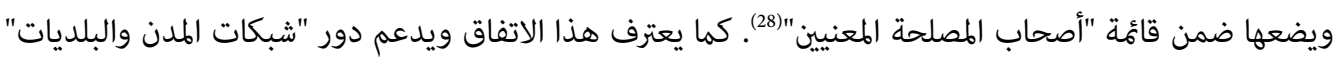
المضيفة للاجئين، ويشجعها على تبادل الخبرات والمهارسات الجيدة والنهج المبتكرة للاستجابات في البيئات الحضرية. كما يدعم ترتيبات التوأمة (29). وتوازيًا مع ذلك، يعترف أيضًا بدور منظمات المجتمع المدني في تحديد مواطن القوة والاحتياجات والتخطيط وبناء القدرات وتنفيذ البرامج وغيرها (30).

ورغم كل ما أشرنا إليه، فإن عديد الجهات المعنية بالتعاون اللامركزي في هذا المجال لا تزال تعتبر أن الاعتراف الدولي به منقوصًا، ويحتاج إلى مزيد من التأكيد والوضوح، وتحديد دقيق لدور هذا التعاون ومكانته (31. لكن الاعتراف التهاف

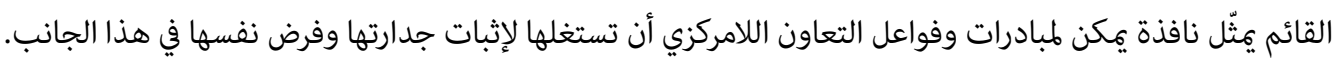

\section{ثانيًا: المساهمات الممكنة للتعاون اللامركزي في مجال هجرة اللجوء}

مل يكن الاعتراف بدور التعاون اللامركزي في التعامل مع الهجرة الدولية، بشكليها الإرادي والقسري، بسبب خصائصها المذكورة ودخولها ضمن نطاق "سيادة" الجماعات المحلية وسلطتها فحسب، بل بدرجة أكبر بسبب طبيعة المساهمة التي يمكن أن يقدمها هذا الشكل من التعاون، وهي مساهمة نوعية وتستجيب لعديد التحديات التي تطرحها هذه بهاه

$$
\begin{aligned}
& 23 \\
& 24 \\
& 25 \\
& 26 \\
& 27 \\
& 28 \\
& 29 \\
& 30
\end{aligned}
$$

31 Eva Dick \& Jana Kuhnt, "The Neglected Role of Cities in the Global Compact on Refugees," The Current Column, German Development Institute (January 2019), accessed on 5/2/2021, at: https://bit.ly/3q6Es5S 
الهجرة عن طريق حلول مبتكرة ومستدامة. نستعرض هنا أبرز المساهمات الممكنة لهذا الشكل من التعاون في مجال تسيير هجرة اللجوء بناء على بنيته المفهومية والنظرية، وعلى تجارب تدخّله السابقة في عدة مناطق من العالم.

\section{1. جمع الشركاء وأصحاب المصلحة وتنسيق جهودهم .}

من بين أبرز خصائص التعاون اللامركزي كونه تعاونًا "متعدد الفواعل"؛ فهو إن كان متمحورًا حول "السلطات المحلية اللامركزية"، فإنه يجمع حولها عديد الفواعل من طبيعة ومستويات مختلفة (سلطات مركزية ولامركزية، ومنظمات

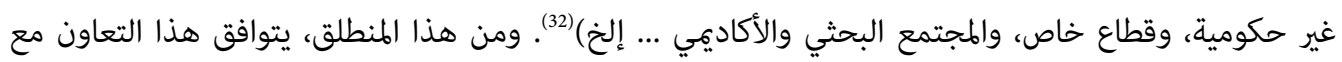

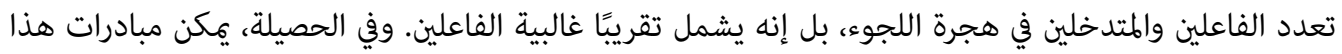

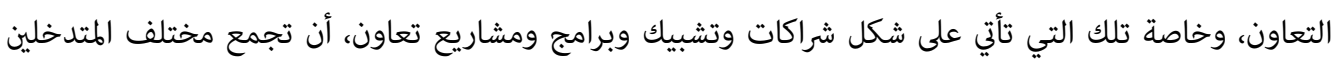

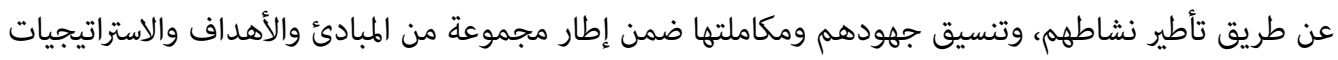

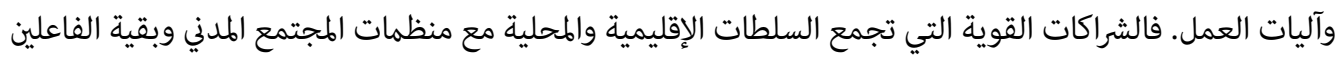

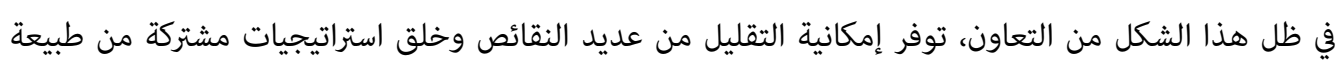

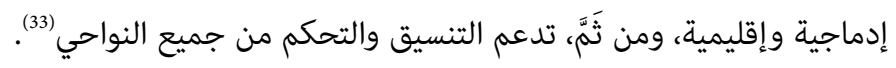

كما أن شبكات التعاون اللامركزي وبرامجه يمكنها أن تمثّل إطارًا مؤقتًا يضبط علاقة السلطات المركزية بالسلطات

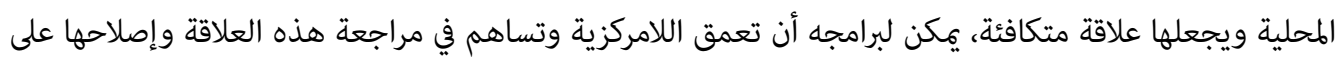

المديَين المتوسط والبعيد.

يتيح هذا النوع من التعاون التنسيق المحلي والأفقي العالي المهم لبلوغ المجموعات الأكثر هشاشة(34) في حالات اللجوء وغيرها، وهو بذلك يساهم في التعامل مع انشغال تعدد الفاعلين المتدخلين الذي عادة ما يشتت الجهود والأموال، ويزعج السلطات المركزية والمحلية في المجتمعات المضيفة للاجئين، ويحرم بعض الفئات من السكان واللاجئين من الحصول على المساعدة والتكفل المطلوب.

\section{2. بناء قدرات الفاعلين وأصحاب المصلحة}

يقدم التعاون اللامركزي في مثل هذه الحالات خدمة مهمة جدًا، تتمثل في بناء قدرات الفاعلين المحليين وأصحاب المصلحة في المجالات التي تحتاج إليها الإدارة الجيدة ملفات الهجرة القسرية، خاصة ما يتعلق بالجانب الإحصائي

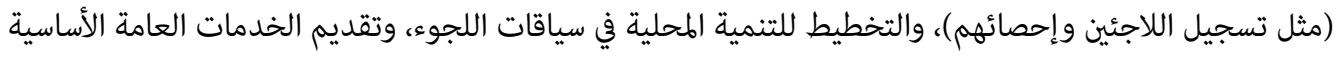
والأشد تضررًا (المياه، الكهرباء، النظافة وغيرها)، وبعث المشاريع الصغيرة ومشاريع البنية التحتية، وتعبئة القطاع ولتهيط الخاص وأصحاب المصلحة. ويقدم التعاون اللامركزي هذه المساهمة عن طريق حزمة من الأدوات، أهمها ورش وشئ وتعاه

32 ينظر تفاصيل عن مقاربات التعاون اللامركزي وكيف تقثّل السلطات المحلية فاعلًا محوريًا يمارس دور القيادة لباقي الفواعل المحليين والناشطين محليًا،

في: مباركية، ص 12-13.

33 Agustí Fernández de Losada Passols, "Shaping a New Generation of Decentralised Cooperation for Enhanced Effectiveness and Accountability," PLATFORMA \& Conference of Peripheral Maritime Regions (CPMR) (October 2017), p. 33, accessed on 5/2/2021, at: https://bit.ly/3jAyYh5

34 Muggah \& Adriana, p. 12. 
العمل والتدريب، والندوات. ويعدّ بناء القدرات من أهم الإسهامات التي يقدمها التعاون اللامركزي للفاعلين

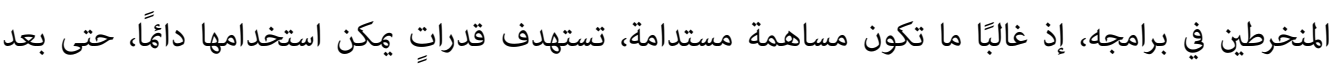
تجاوز حالات الأزمات.

\section{3. تشارك التجارب والممارسات الجيدة}

يقدم الفاعلون في إطار التعاون اللامركزي واحدة من الخدمات الأساسية، هي تشارك الخبرات والتجارب فيما بينهم. وهجرة اللجوء، من بين المجالات التي تستوجب مثل هذه المساهمة؛ فحتى مع الطبيعة الحضرية والإقليمية لهذه

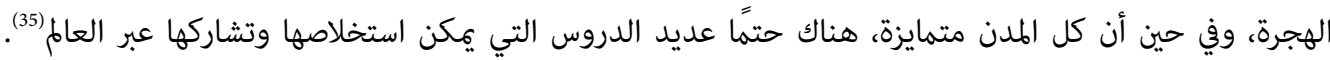
وتزداد قيمة هذه المساهمة، بالنسبة إلى البلديات التي تفتقر إلى خبرة أو تجربة سابقة في استضافة اللاجئين، وتلك الك

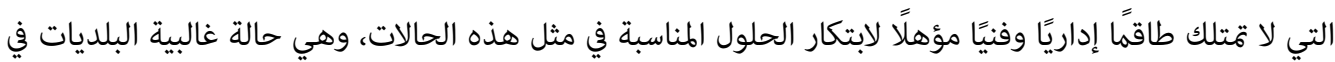
الدول النامية وفي منطقة الشرق الأوسط وشمال أفريقيا. وقد تحفظت عديد الدراسات والتقارير على كون المدن غالبًا ما تعمل بكعزل عن بعضها البعض، في الوقت الذي

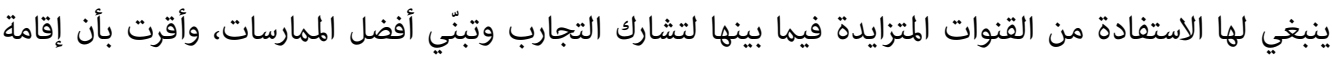
الشراكات بين المدن في الدول النامية وإدماجها في شبكات ما بين المدن سيساهمان في ترقية التنمية، وتكييف حلول مبتكرة وتبنّيها؛ للتقليل من أخطار تدفقات اللاجئين إليها وتعظيم مزاياها

\section{4. دفع التحول نحو المقاربة التنموية}

يساهم التعاون اللامركزي من خلال مبادراته المختلفة، باعتباره شكلًا من أشكال التعاون التنموي الدولي، في دعم تحوّل مقاربة التعامل مع حالات اللجوء الواسعة النطاق والممتدة من التعامل الإنساني (الإغاثة والمساعدة

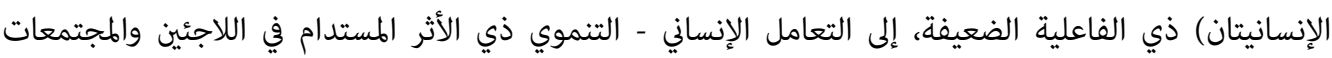
المضيفة على حد سواء. فمن خلال منطق المشاريع والمشاريع المصغرة الذي يقوم عليه، يساهم هذا التعاون في جعل

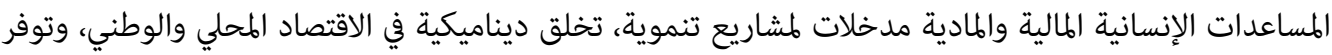
فرص عملٍ ودخلاً مستدامًا للاجئين والمجتمعات المضيفة، وبناء عليه، يفك تبعيتهم للمساعدة الإنسانية، ويكرس استقلاليتهم ويثمن رأس مالهم البشري.

\section{5. التمويل المستدامم للاستجابات الإنسانية - التنموية}

على الرغم من أن التعاون اللامركزي ليس تحويلًا أو تقديًا للمساعدة الهالية المباشرة، فإنه جاء أساسًا لتدارك نقائصها

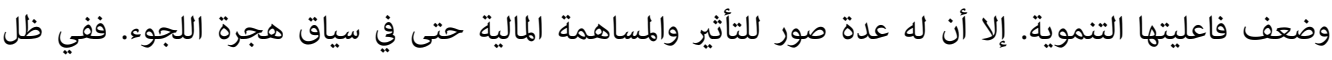
الحاجة المالية للسلطات المحلية، يساهم هذا الشكل من التعاون في المساعدة على إيجاد حلول مبتكرة لا تحتاج 
إلى كثير من المال، وخاصة بالتنسيق مع المنظمات غير الحكومية المعروفة بنجاعتها من الناحية المالية. ومن جهة أخرى، ومن خلال التجارب الجيدة القابلة للنقل، والمساعدة الفنية على نقلها وتكييفها على النحو المناسب، يساهم التعاون اللامركزي في توفير كثير من الجهد والمال بالنسبة إلى البلديات المستفيدة، كما يكنه أن يكون مصدرًا للتمويل البسيط والمستدام لاستجابات مختلف الفاعلين في مجال هجرة اللجوء، وذلك عن طريق مشاريعه وبرامجه المتوسطة والبعيدة المدى التي تقوم على أساس اتفاقيات مالية تسمح بتمويل بسيط، لكنه مستدام نوعًا ما. وإذا نظرنا إلى أزمة اللجوء الحالية التي جاءت في ظل أزمات وصعوبات اقتصادية لدول أوروبا ودول الشرق الأوسط وشمال أفريقيا، تتعزز قيمة مبادرات التعاون اللامركزي التي تعتمد على تمويل قليل وذي فاءئ فائدة كبيرة.

\section{6. محْللة الأطر والاتفاقات الدولية لهجرة اللجوء وتوجيه الفاعلين}

عرف التعاون الدولي في مجال الهجرة واللجوء تطورًا مهمًا مع اعتماد الاتفاقين العالميين للهجرة واللجوء، وإضافة إلى مساهمة عديد فاعلي التعاون اللامركزي (منظمات وشبكات) في مناقشات صياغة تلك المواثيق واعتمادها، والنضال

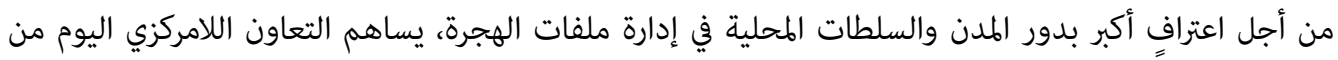

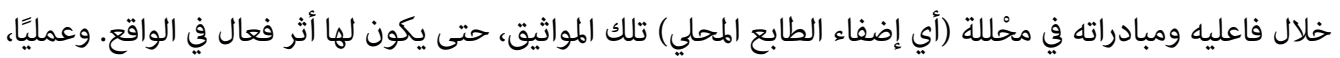

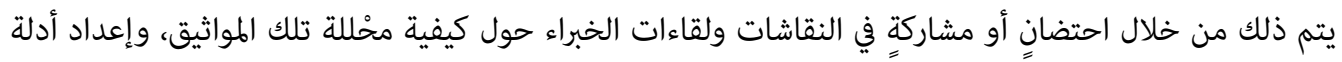

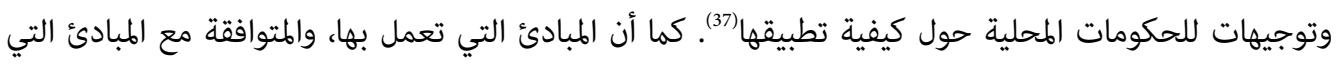
قامت عليها المواثيق الدولية، تتحول إلى مبادئ مرشدة ومؤسسة لمبادرات تعاون أخرى، بل لأجندات التعات التعاون

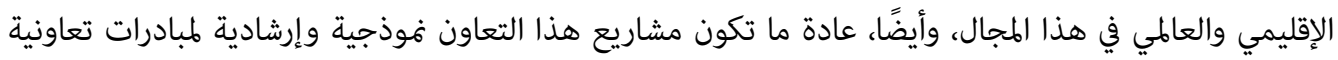
أخرى. وتسمح برامج التعاون اللامركزي، من خلال عدة نشاطات تتضمنها، بوضع معايير تنفيذية تمثّل مرجعية للتعامل مع مثل هذه الأزمات والحالات (38).

ويساهم أيضًا نضال المنظمات وشبكات الفاعلين المحليين في جعل الهجرة ضمن أجندات بعض الإدارات المحلية التي

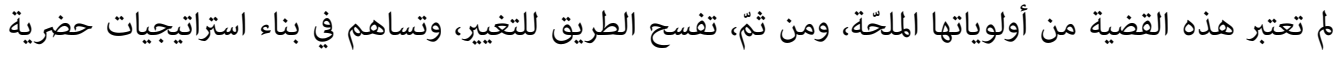
واقتصادية خاصة بالمخيمات والمدن المضيفة للاجئين.

ومع أهمية كل المساهمات المذكورة، فإن الأهم أن ما يقدمه التعاون اللامركزي عمومًا، خاصة عندما يأتي على شكل

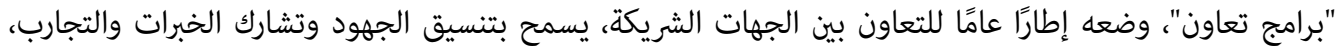
والتدريب وبناء القدرات ذات الفائدة والاستخدام المستدام الذي يعزز صمود المدن والبلديات المضيفة للاجئين في الفترة

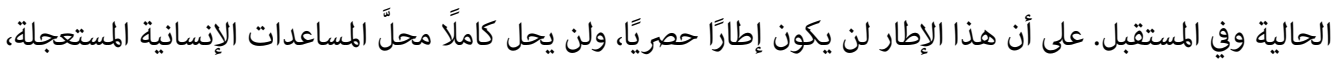
ولا تدخلات الحكومات المركزية والمحلية وسياساتها، وإما يكملها، ويتجه بها نحو مزيد من الفاعلية والكفاءة والاستدامة.

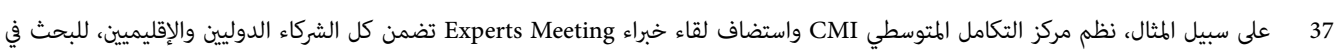

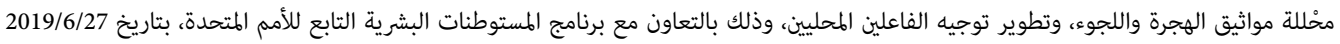

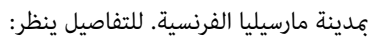
"Localizing the Global Compacts: UN Experts Meeting," Center for Mediterranean Integration (CMI), 27/6/2019, accessed on 28/1/2020, at: http://bit.ly/2My2Az4

$38 \quad$ Muggah \& Adriana, p. 13. 


\section{ثالثًا: انشغالات المدن العربية المضيفة للاجئين واحتياجاتها}

في ظل أزمة اللجوء الراهنة، يُتوقع أن يكون هناك كثير من البلديات لمواجهة هذه الأزمة، ولكن مل يتم تزويدها سوى بقليل من الدعم والتوجيه لساعدتها على التكيف مع عديد المسائل ذات الصلة، مثل: تسجيل القادمين، والحفاظ على الأمن، وضمان قدر مناسب وكافٍ من المياه والكهرباء وخدمات الصرف الصحي، وتهي، وتسهيل الحصول على مأوى، والتوسط والوقاية من التوتر بين الجماعات، وذلك كله جزء صغير من الضغوطات الكثيرة التي تتعرض مهن

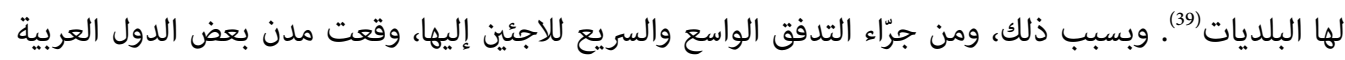

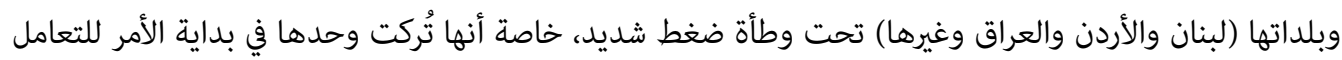

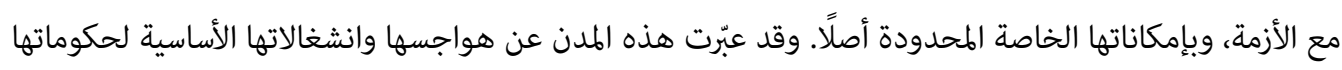
الوطنية وللأطراف المعنية في المجتمع الدولي، ودعت إلى التضامن والتعاون معها لإيجاد حلول للأزمة.

يتطلب تحديد أطر التعاون المطلوبة وأشكاله، وتنظيم استجابات فعالة ومستدامة لأزمة لجوء بالحدة والخصائص المذكورة، أولًا تشخيص احتياجات الأطراف المعنية، ولا سيما في هذه الحالات احتياجات البلديات والسلطات المحلية من جهة، واحتياجات المجتمعات المضيفة واللاجئين من جهة أخرى. وهو الأمر الذي عملت عليه عديد الجهات المتدخلة في أزمة اللاجئين الجارية.

وعلى الرغم من تعدد محاولات التشخيص والطسوح التي أُجريت، فإننا لاحظنا أن نتائجها متقاربة إلى حد بعيد على

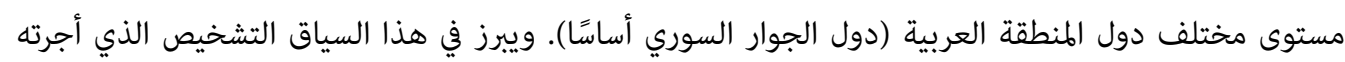
مؤسسة "ميرسي كوربس/ فيالق الرحمة" Mercy Corps في لبنان عام 2014، والذي سيُتخذ مرجعًا، بكعية نتائج استطلاعات "شبكة التعلم للبلديات المضيفة" التابعة لـ "برنامج اللاجئين والمجتمعات المتوسطية المضيفة"، لتحديد الاحتياجات العامة، وتصور الاستجابات المطلوبة والتأطير اللازم لها.

حاجات اللاجئين الحضريين معروفة، غالبًا، من حيث النوع (الخدمات الأحيائية العامة: السكن أو الملجأ، والهاء،

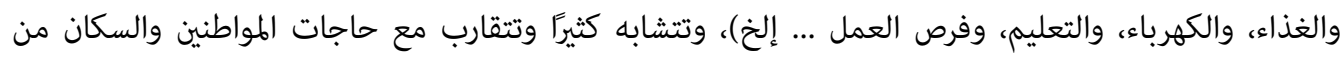

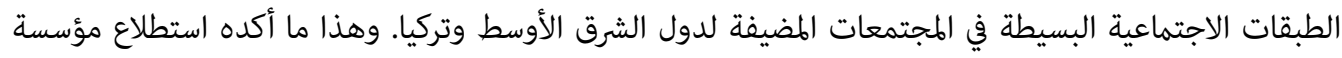

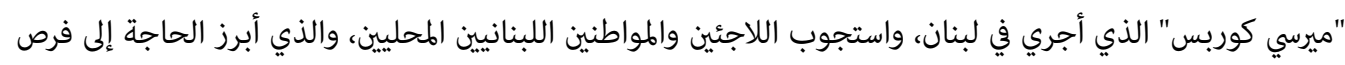

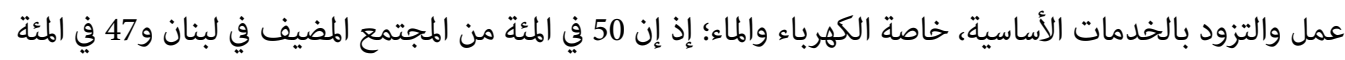

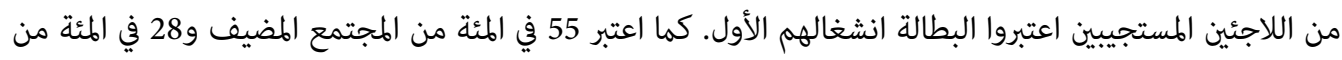
اللاجئين المستجيبين أن الكهرباء حاجة ماسة وغيابها أو اضطرابها ميّّل مشكلة حقيقية. واعتبر 36 في المئة من السكان

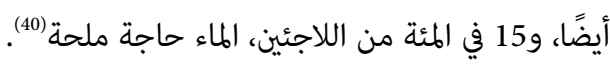

والملاحظ أن هذه الحاجات تتعلق بمجموعة من الخدمات العامة المحلية التي تدخل ضمن صلاحيات السلطات المحلية للبلديات ومهماتها، وتتسبب في زيادة الضغط على منظومة تقديم تلك الخدمات بفعل طلب اللاجئين 


\section{شكل يوضح الأولويات الأساسية للبلديات اللبنانية المضيفة للاجئين في مجالات بناء القدرات}

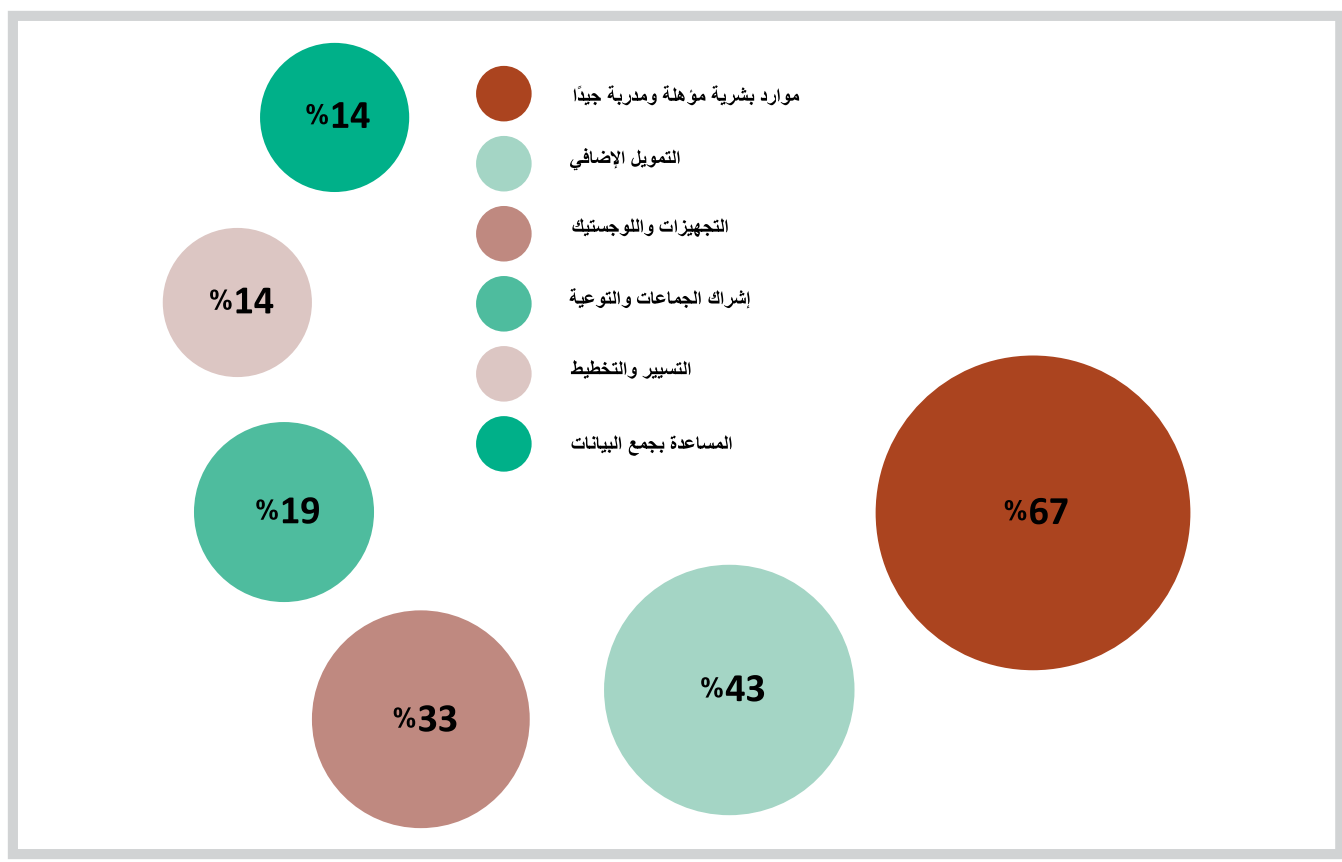

المصدر:

"Engaging Municipalities in the Response to the Syrian Refugee Crisis in Lebanon," Policy Brief, Mercy Corps (March 2014), p. 14, accessed on 5/2/2021, at: https://bit.ly/3rwGnkf

الحضريين عليها. ومن أجل دعم منظومة خدماتها، ومساعدتها على الصمود، والتكفل باللاجئين والمجتمع المحلي في إطار استجابة تنموية متكاملة، تبلورت لدى السلطات المحلية مجموعة من الاحتياجات. يستعرض الشكل الحالة اللبنانية، ويليه شرح وتوضيح لمختلف الحاجات المعبّر عنها.

\section{1. الحاجة إلى التدريب وبناء القدرات}

ليس من المفاجئ اعتبار أن البلديات تحتاج إلى دعم وبناء أكثر لقدراتها، بالنظر إلى الزيادة الحادة في السكان

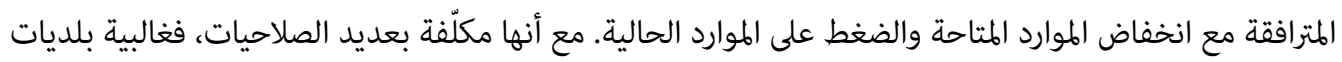

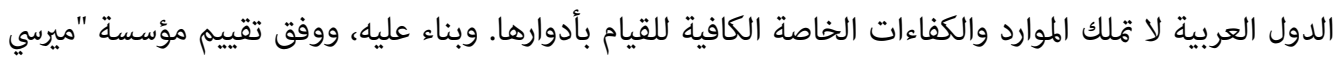

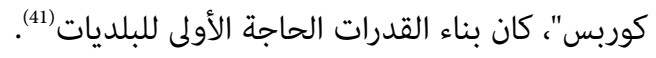

المدن مسؤولة أولًا عن تقديم الخدمات العامة لكل السكان الحضريين، وفي سياقات اللجوء، ينبغي لها تقديم

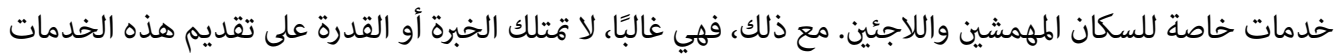

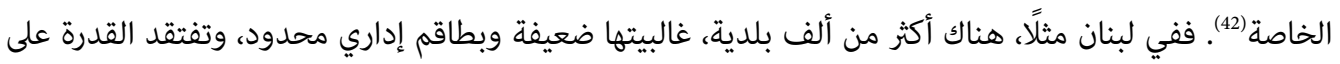

41 Ibid., p. 12.

42 "Urban Refuge: How Cities Are Building Inclusive Communities," p. 15. 
تقديم الخدمات التنموية، وهي أوجه قصور تسبق الأزمة السورية(43)، ولكنها تفاقمت معها. كما أن غالبية البلديات لا تعرف بنية المانحين والتنسيق الإنساني، وسيكون عليها الاطلاع على ذلك من أجل تنسيق جيد مع بقدئ بقدية الفاعلين (44). ويتوافق هذا مع نتائج استطلاع لـ "برنامج اللاجئين والمجتمعات المتوسطية المضيفة"، فقد أكد 97 في المئة من ممثلي البلديات المستجيبة حاجتهم إلى مزيد من الدعم الفني والتدريب وبناء القدرات في مجالات إدارة الأزمات، والتزويد بالخدمات في مختلف القطاعات (خاصة المياه والبنية التحتية) (45).

\section{2. الحاجة إلى التعلم والاستلهام من التجارب}

موجات لجوء بهذا الحجم والنوع والسرعة، لا يمكن التعامل معها ارتجاليًا أو مؤقتًا، وإفا تستدعي تعاملًا مبتكرًا ومدروسًا ورصينًا، ومجربًا أيضًا. ولكن الموظفين البلديين والطمثلين المنتخبين الذين يبحثون عن الابتكار والحلول

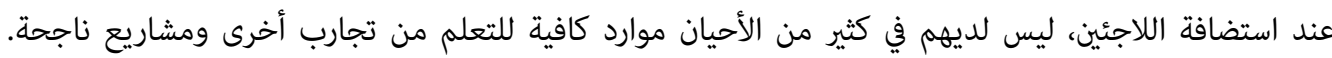

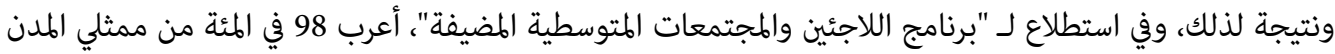
والبلديات المستجيبة وموظفيها عن اهتمامهم ورغبتهم في تبادل التجارب مع البلديات الأوروبية المضيفة (46).

\section{3. الحاجة إلى التمويل الإضافي والمستداهم}

أرهقت أزمة اللاجئين حكومات الدول المضيفة وبلدياتها التي هي أصلًا دول متوسطة الدخل، وجماعاتها المحلية

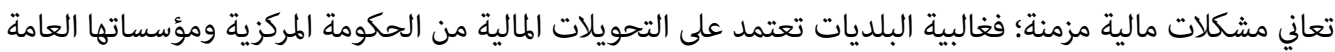

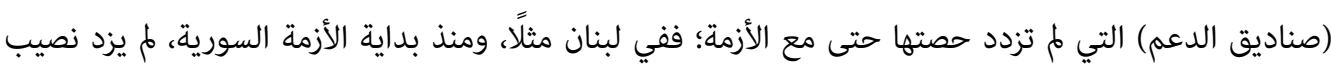

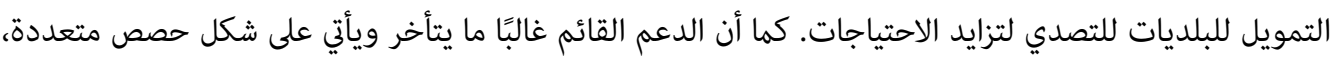
ما يعيق قدرة البلديات على وضع مخططات ومشاريع طويلة الأمد (47).

زادت الأزمة الحالية من حاجة الدول والبلديات المضيفة إلى التمويل والاستثمار من أجل توسيع قطاعات الخدمات

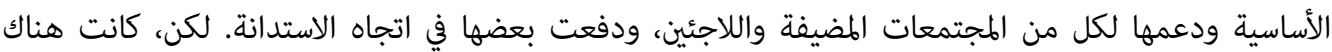

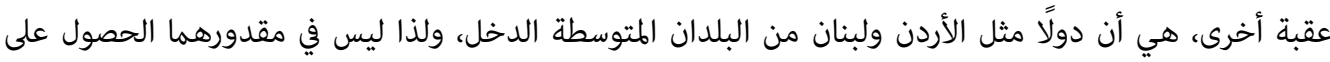

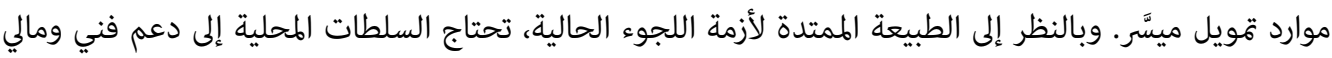
مستعجل ومستدام، حتى تتمكن من وضع أنظمة للتعامل مع حاجات المجتمعات واللاجئين في السنوات القادمة (48)،

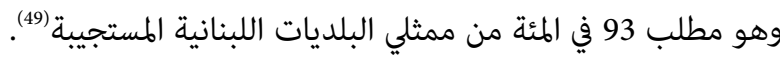

43 Dima Mahdi, "Understanding Refugee Politics in Lebanon and Calls for Repatriation," LCPS Policy Briefs, no. 30, The Lebanese Center for Policy Studies (October 2017), p. 5, accessed on 5/2/2021, at: https://bit.ly/3tFHGPC

44 "Engaging Municipalities in the Response to the Syrian Refugee Crisis in Lebanon," p. 12.

45 "Mediterranean Refugees and Host Communities Program," Center for Mediterranean Integration, Amman Peer to Peer Learning Workshop (Presentation), 31/5/2016, p. 8, accessed on 1/2/2021, at: https://bit.ly/3psRnhV

46 Ibid.

47 "Engaging Municipalities in the Response to the Syrian Refugee Crisis in Lebanon," p. 13.

48 Ibid., p. 20.

49 Ibid., p. 23. 
تعتقد البلديات أن التدارك يأتي بإصلاح ماليّة البلديات على المدى المتوسط والبعيد، وهذا سيتطلب تغييرات في القوانين

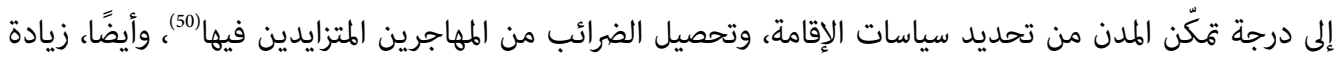
الدعم المالي فورًا من السلطة المركزية، وعن طريق التعاون المحلي (تعبئة القطاع الخيري والقطاع الخاص) والدولي.

\section{4. الحاجة إلى التجهيز ودعم نُشاطاتها}

عبرت بعض البلديات عن حاجتها العاجلة إلى تجهيزات بعينها لدعم نشاطاتها، مثل: جمع النفايات (حاويات وعربات جمع القمامة)، والتزويد بالمياه (شبكات توزيع وصرف اجلهات المياه) (51).

\section{5. الحاجة إلى التنسيق مع المتدخلين الآخرين وبينهم}

من السهل التكهن بأنه في ظل وجود قيادة مركزية ضعيفة من طرف الحكومات المحلية، يكن أن تعاني المساعدات

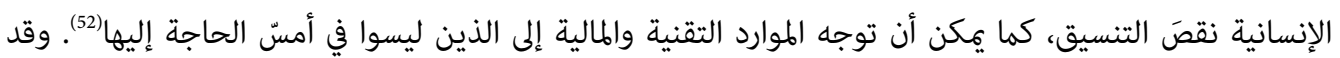

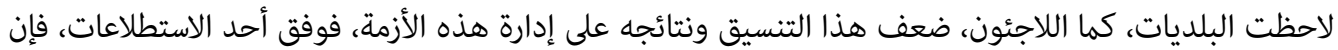

51 في المئة من اللاجئين المستجيبين في لبنان أكدوا ضعف التنسيق بين المنظمات والبلديات واشتكوا منه (53.

وفي ظل الاعتراف بأن مزيدًا من التنسيق ما بين المنظمات سيساعد على تحديد الحاجات، ويزيد من درجة التكاملية

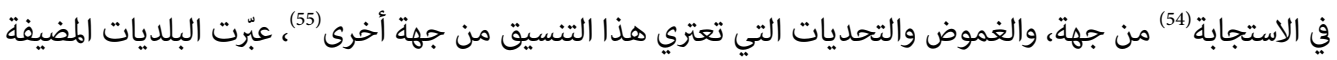

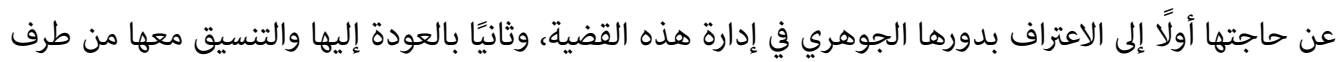

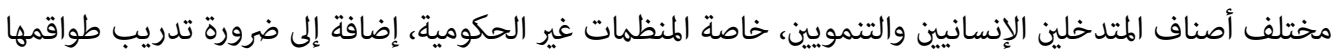
على إقامة الشراكات والميكانيزمات المطلوبة لتفعيل هذا التنسيق.

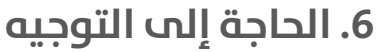

من شأن وجود أطر وطنية أن يوجه البلديات وأجهزة الأمن إلى تبنّي مقاربات سياساتية تجاه اللاجئين على المستوى

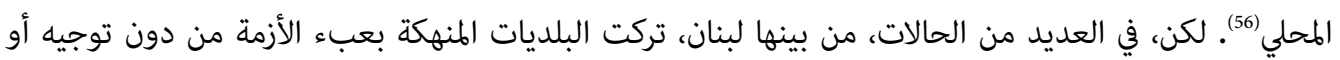

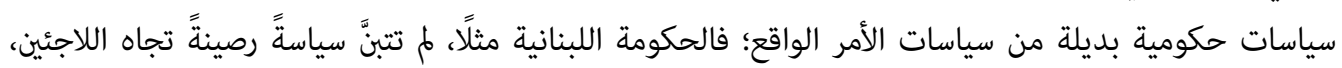

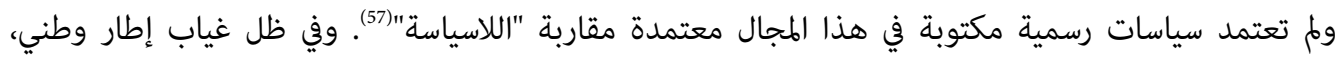

$50 \quad$ Muggah \& Adriana, p. 11.

51 "Engaging Municipalities in the Response to the Syrian Refugee Crisis in Lebanon," p. 14.

52 Silvia Ciacci, "The Partnership with Local Authorities in Responding to Humanitarian Crisis: The Case of Lebanon, Lessons learned and Recommendations, Part Two," Oxfam Italia (September 2014), p. 27, accessed on 5/2/2021, at: https://bit.ly/36SEQNC

53 "Engaging Municipalities in the Response to the Syrian Refugee Crisis in Lebanon," pp. 16, 17.

54 Francesca Grandi, Kholoud Mansour \& Kerrie Holloway, Dignity and Displaced Syrians in Lebanon: 'There is no Karama here' (London: Humanitarian Policy Group; Overseas Development Institute, 2018), p. 25.

55 "Urban Refuge: How Cities Are Building Inclusive Communities," p. 6.

56 Mahdi, p. 1.

57 Ibid., p. 5. 
فإن سياسات اللاجئين، بحكم الواقع، تمليها البلديات والأجهزة الأمنية التي فتحت الباب لاعتماد مبادرات، خارج

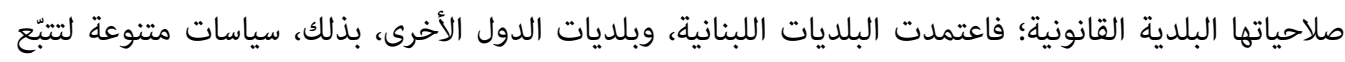
حركة اللاجئين وضبطها (58). لكن العديد منها مؤقت وغير مضمون النتائج، ويفتقر إلى البيانات الدقيقة، وهو ما جعل المدل البلديات المعنية تؤكد حاجتها إلى التوجيه نحو السياسات المناسبة.

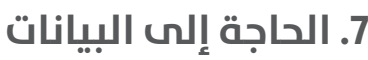

بالنظر إلى ضعف أنظمة الإحصاء الوطنية والمحلية، أو شبه غيابها في مسائل الهجرة في عديد دول المنطقة، وبالنظر

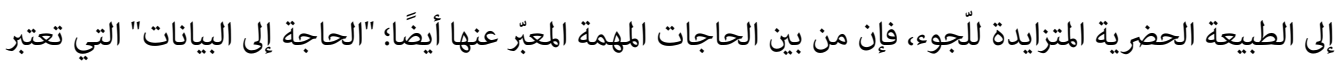

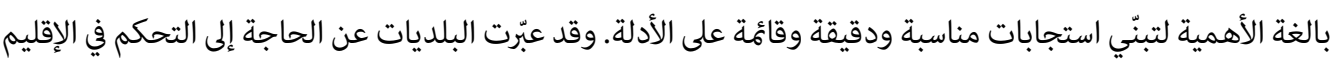
ومعرفة أين يقيم اللاجئون (59)، وأن أحد القيود الأساسية أمامها هو الافتقار العام إلى البيانات الخاصة باللاجئين (60).

\section{رابعًا: برنامج اللاجئين والمجتمعات المتوسطية المضيفة}

استنفرت أزمة اللجوء الجارية كثيرًا من الأطراف المعنية، وأثارت استجابات عديدة من طبيعة مختلفة. وتركيزًا

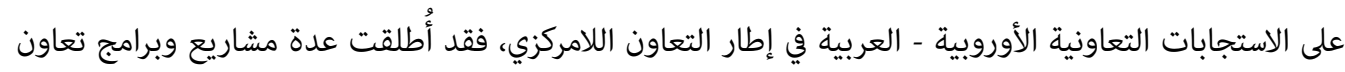

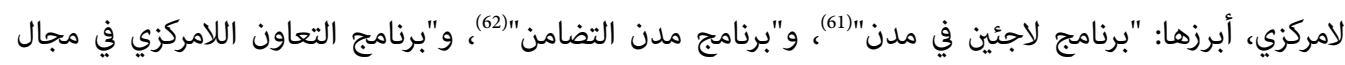

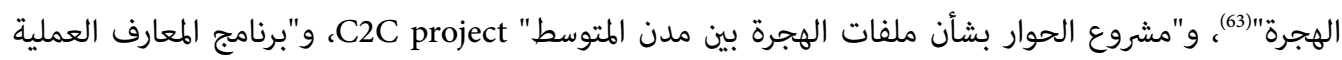

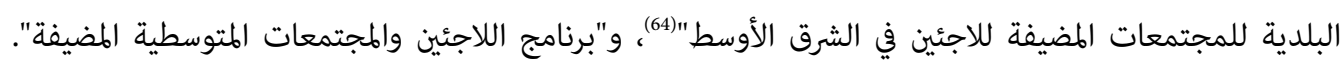

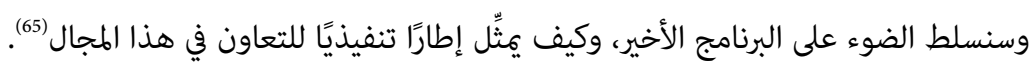

\section{1. التعريف بالبرنا مج وأهدافه العامة}

"برنامج اللاجئين والمجتمعات المتوسطية المضيفة"(66) هو برنامج إقليمي للتعاون اللامركزي في مجال هجرة اللجوء بين الدول (السلطات المحلية) الأوروبية المتوسطية والسلطات المحلية لبعض دول الشرق الأوسط وشمال أفريقيا، أُطلق في سياق أزمة اللجوء التي ألّت بالمنطقة عقب الأزمة السورية، ليغطي الفترة 2016-2021، ويساهم في تشارك

\footnotetext{
$58 \quad$ Ibid.

59 Ciacci, p. 26.

60 Mahdi, p. 5.
}

61 61 ينظر الموقع الإلكتروني للبرنامج: Refugees in Towns, accessed on 5/2/2021, at: http://bit.ly/3oyZF6V.

62 ينظر الموقع الإلكتروني للبرنامج: Solidarity Cities, accessed on 5/2/2021, at: https://bit.ly/36FrIex.

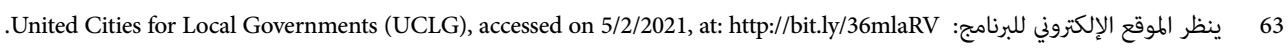

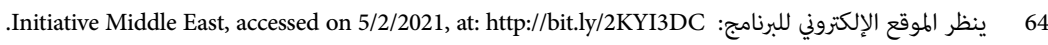

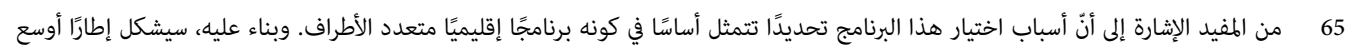

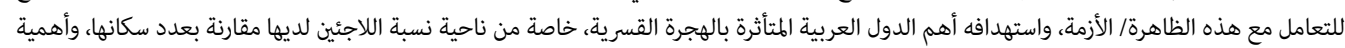

الفواعل وأصحاب المصلحة المنخرطين في البرنامج، وتنوع طبيعتهم.

66 يستضيف موقع مركز التكامل المتوسطي صفحة البرنامج، ينظر: Conimarseille, accessed on 28/1/2020, at: https://bit.ly/3abJdUP. 
المسؤولية وتخفيف العبء على المدن والمجتمعات المضيفة للاجئين في المنطقة، وتحفيز التحول في نهج التعامل مع أوضاع الهجرات القسرية الممتدة، واعتماد مقاربة تنموية في هذا المجال.

ويشمل هذا البرنامج الدول والبلديات المضيفة في الشرق الأوسط وتركيا وشمال أفريقيا وأوروبا. ويركز في منطقة

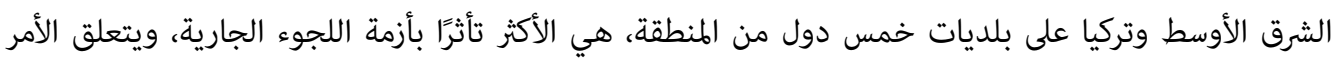

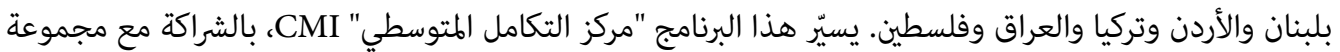
من الفاعلين المهمين في هذا المجال، وهم على وجه التحديد: الوكالة الفرنسية للتنمية AFD، ووكالة التنمية الدولية الألمانية GIZ، وشبكة مدن المتوسط Medcities، ومجموعة الأمم المتحدة، والمنظمة الدولية للهجرة IOM، والمركز الدولي لتطوير سياسات الهجرة ICMPD، ومنظمة مدن وحكومات محلية متحدة/ فرع الشرق الأوسط وغرب آسيا UCLG MEWA

ويتمثل الهدف العام من هذا البرنامج في تقوية صمود المجتمعات المضيفة واللاجئين، وتحسين قدرتهم الاستجابية للهجرة القسرية بالتركيز على الاحتياجات التنموية المتوسطة الأمد لكليهما. وينقسم هذا الهداف الهدف ثلاثة أهداف فرعية،

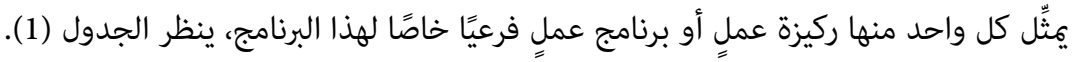

\section{2. المساهمات العملية للبرنامج في التعامل مع أزمة اللجوء}

قام هذا البرنامج، خلال ثلاث سنوات منذ إطلاقه، بعديد المبادرات والتدخلات التي تمسح مختلف برامج عمله

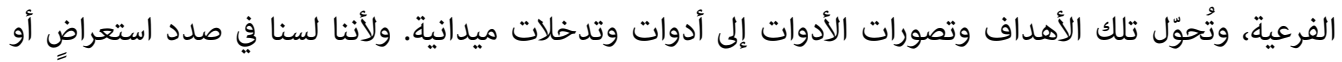
تقييم لتدخلات هذا البرنامج فحسب، وإفا لتوضيح كيفية تشكيله بوصفه إطارًا تنفيذيًا للتعاون في هذا لهاب المجال، ومدى استجاباته لانشغالات المدن المضيفة المحددة في المحور السابق، فلن نستعرض تحركاته على لته أساس أركان العمل وبرامجه المشار إليها، وإما بحسب استجابتها لحاجات المدن المضيفة المعبّر عنها. 
(1) الجدول

البرامج الفرعية لبرنامج اللاجئين والمجتمعات المتوسطية المضيفة

\begin{tabular}{|c|c|c|c|}
\hline \multicolumn{3}{|c|}{ تفاصيل البرامج الفرعية } & \multirow{2}{*}{ الفرنامج الفرعي } \\
\hline 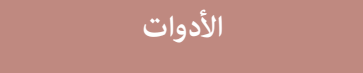 & الهدف & محور العمل & \\
\hline 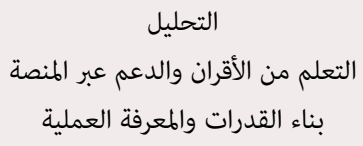 & المقليفيز قدرة استجابة الحكومات المحلية & قدرات المجتمعات المحلية التي & 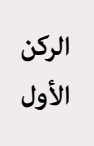 \\
\hline $\begin{array}{c}\text { إشراك النساء والشين الفاعلين } \\
\text { التحباب }\end{array}$ & 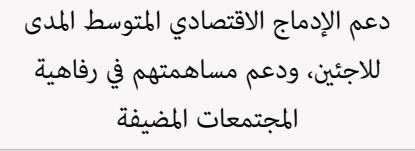 & إدماج اللاجئين من أجل الرفاه المشترك & 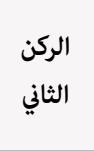 \\
\hline $\begin{array}{c}\text { النقاش بين الأطراف المعنية } \\
\text { التشبيك بين الدياسبورا }\end{array}$ & 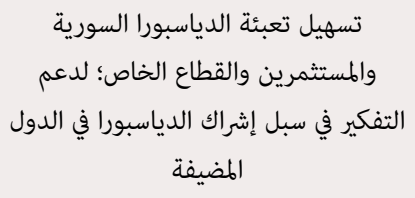 & تعبئة الشتات السوري (الدياسبورا & 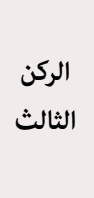 \\
\hline
\end{tabular}

المصدر: من إعداد الباحث استنادًا إلى محتوى مطوية البرنامج:

Center for Mediterranean Integration, Mediterranean Refugees and Host Communities Program: 2016-2021, accessed on 21/2/2021, at: https://bit.ly/3bm5HDa

\section{أ. جهود دعم التعلمم وتعزيز قدرات البلديات المضيفة}

يعتبر بناء قدرات المجتمعات المضيفة واللاجئين من أجل الحفاظ على رفاهيتهما في سياق الأزمة، وحتى بعدها، الركن الأول لعمل هذا البرنامج، وتتوافق أهميته مع أهمية هذا المطلب لدى البلديات المضيفة، مثلما أوضحنا في المحور السابق.

وارتكزت هذه الجهود أساسًا على إنشاء شبكة تعلم تضم مختلف الأطراف المعنية تحت مسمى "شبكة تعلم البلديات المتوسطية المضيفة" HMLN، وتزويدها بمنصة للتبادل الإلكتروني، تسمى "التنسيق من أجل التنمية" C4D

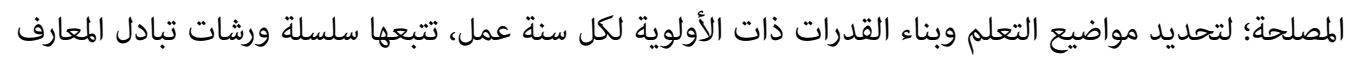
والتجارب والتدريب والندوات والمناقشات الإلكترونية. ومنذ إطلاقها في حزيران/ يونيو 2016، نظّمت "شبكة تعلم البلديات المتوسطية المضيفة" عديد الفعاليات لدعم الحكومات المحلية المضيفة بالمعرفة القائة على الأدلة وبناء القدرات؛ لتحسين القدرة على الاستجابة للتهجير القسري. وقد استفادت الفواعل المنخرطة في الشبكة من عدة ورش عمل وتدريب فعلية وندوات افتراضية حول المواضيع

68 ينظر رابط المنصة التي يحتضنها البنك الدولي باعتباره شريكًا لمركز التكامل المتوسطي في "برنامج اللاجئين والمجتمعات المتوسطية المضيفة": Collaboration for development, accessed on 5/2/2021, at: http://bit.ly/3ptArrA 
ذات الأولوية، كما شاركت في الاستبيانات، وكذلك المناقشات عبر الإنترنت وعبر المنصة التعاونية المخصصة لذلك،

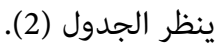

\section{الجدول (2)}

حصيلة نشاطات "برنامج اللاجئين والمجتمعات المتوسطية المضيفة" خلال الفترة 2017-2019

\begin{tabular}{|c|c|c|c|c|}
\hline \multirow{2}{*}{ المجموع أل الموع } & \multicolumn{3}{|c|}{ تاريخ التنظيم } & \multirow{2}{*}{ 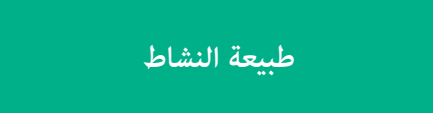 } \\
\hline & 2019 & 2018 & 2017 & \\
\hline 5 & 1 & 3 & 1 & استطلاعات (مسوح) الاحتياجات والأولويات \\
\hline 3 & 1 & 1 & 1 & لقاء سنوي مباشر \\
\hline 10 & 3 & 4 & 3 & ندوات إلكترونية مواضيعية \\
\hline 2 & 0 & 1 & 1 & خلاصات تجارب وممارسات جيدة \\
\hline 4 & 1 & 1 & 2 & ورشات عمل \\
\hline 2 & - & 2 & - & زيارات ميدانية \\
\hline 8 & 3 & - & 5 & حضور، ومشاركة في نشاطات الشركاء \\
\hline 3 & 1 & 2 & - & جوائز ائز \\
\hline
\end{tabular}

المصدر: من إعداد الباحث استنادًا إلى المصادر المختلفة لـ "برنامج اللاجئين والمجتمعات المتوسطية المضيفة".

وقد برزت "التنمية الاقتصادية المحلية" LED في سياقات الهجرة القسرية، بوصفها موضوعًا ذا أولوية لنشاط الشبكة التعليمي والتدريبي، وذلك باعتبارها حلًا مستدامًا يتوافق وخيار الحل الإدماجي، وكذلك كونها تصبّ في مصلحة المجتمعات المضيفة واللاجئين على حد سواء، وتؤسس لصمود طويل المدى على مستوى البلديات المشمولة.

كما شملت جهود التدريب وتعزيز القدرات مجالات خدماتية مهمة، نحو المياه والنظافة والصحة والتعليم، وغيرها، ويمكن الوصول إلى تفاصيل هذه النشاطات والمستفيدين منها وتقييمهم لها عبر رابط البرنامج على موقع مركز التكامل المتوسطي المشار إليه سلفًا.

كما عملت شبكة التعلم، على نحو ممنهج واحترافي، على تجميع أبرز التجارب والطمارسات الجيدة المتعلقة بجوانب مختلفة لإدارة ملف الهجرة القسرية بمقاربة تنموية، وتم تحريرها ونشرها في خلاصتين لتستفيد منها البلديات والمدن المعنية في المنطقة وخارجها. استعرضت الخلاصة الأولى 20 تجربة تغطي مجالات الحوكمة، والتماسك الاجتماعي، والاندماج في سوق العمل، وتسيير النفايات الصلبة، والإسكان والتخطيط الحضري (69). في حين استعرضت الخلاصة الثانية 16 تجربة حول موضوعات: تمكين بيئة الأعمال وتعبئة القطاع الخاص، وتقديم الخدمات العامة، والتعليم والتدريب، والإدماج الاجتماعي، والاقتصاد وخلق فرص عمل محلية (70).

69 Center for Mediterranean Integration, Best Practices in Hosting Refugees (Marseille: CMI, 2017), accessed on 2/2/2021, at: https://bit.ly/3ch97cj

70 Center for Mediterranean Integration, Experiences in Hosting Refugees: Local Economic Development in Host Communities (Marseille: CMI, 2018), accessed on 2/2/2021, at: https://bit.ly/3qHwwbh 
رغم الحضور القوي للنشاط الإعلامي لهذا البرنامج، فإنه غير واضح من الناحية المالية، و"لا يوفر" بيانات بخصوص هذا الجانب. ولكن من خلال تجنيد هذا البرنامج عددًا كبيرًا من الشركاء والمانحين والمنظمات غير الحكومية والقطاع الخاص، فإنه يوفر هامشًا تمويليًا إضافيًا من جهة، ويقلل من تكلفة هندة التدخلات مستفيدًا من مزايا مشاركة المنظهات غير الحكومية من جهة أخرى. وفي صلب هذا السياق، نظم مركز التكامل المتوسطي وشركاؤه، في أيلول/ سبتمبر 2015، مؤتمرًا رفيع المستوى لأصحاب المصلحة حول الأزمة السورية تحت شعار "من الصمود إلى التنمية"(71)، من أجل جلب الدعم المالي للدول

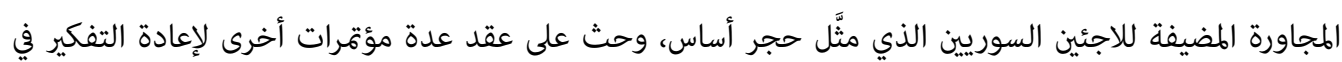
سبل هذا الدعم من أجل الاستجابة للتحديات التنموية البعيدة الأجل، بما فيها صيغ التمويل الميسر، مثل "مؤتمر المانحين لدعم سورية والمنطقة" المنعقد في شباط/ فبراير 2016 بلندن (72). كما أنه بتعبئة الدياسبورا، فإنه يوفر تمويلًا إضافيًا ويخفف العبء المالي على البلديات المضيفة، ويخلق فيها حركية اقتصادية، وينشط الدورات المالية. وقد تجسّد هذا أساسًا من خلال تنظيم منتدى الجالية السورية للأعمال (شباط/ فبراير 2017)، وتسهيل البرنامج للقاءات التشاورية بين رجال الأعمال الدوليين السوريين، ما أدى إلى إنشاء

$$
\text { الجمعية الدولية السورية للأعمال SIBA ومباشرة أعمالها (73). }
$$

\section{ج. المساعدة على جمع البيانات وتشاركها}

بالنظر إلى أهمية البيانات في تكوين استجابات قائة على الأدلة التي أكدتها المواثيق الدولية الخاصة بالهجرة واللجوء(74)، وعبّرت المدن والبلديات المضيفة عن الحاجة إليها، فقد اعتمد البرنامج عدة أدوات لإنتاج المعلومات والبيانات وتشاركها في هذا الشأن، فعلاوة على شبكة التعلم ومنصة التبادل الإلكترونية، فإن للبرنامج نشرة خاصة حول اللاجئين والمجتمعات المضيفة، وفضاء لمدونات القصص والتجارب، إضافة إلى قاعدة بيانات خاصة بالمدن المتوسطية المضيفة للاجئين ... إلخ. وتسمح كل هذه الأدوات بحصول الفاعلين والمهتمين على معرفة محيّنة حول

$$
71
$$

"Syria Refugee Crisis: Supporting Mashreq Countries in their Development Agenda," Center for Mediterranean Integration, 7/9/2015, accessed on 5/2/2021, at: http://bit.ly/3qXi6nc

$$
72 \quad \text { لتفاصيل عن المؤتمر وحيثيات نقاشاته، والمبالغ والوعود المالية التي أسفر عنها، ينظر: }
$$

"Supporting Syria and the Region Donors' Conference in London," Center for Mediterranean Integration, 4/2/2016, accessed on 28/1/2020, at: http://bit.ly/2YkFZcb

73

تدريبية. ينظر:

Burton Bollag (ed.), CMI Annual Report 2018: It's All about Partnership (Marseille: Center for Mediterranean Integration, 2018), p. 36; Janette Uhlmann, "Center for Mediterranean Integration: Perspectives from the Mediterranean Host Municipalities Network," KNOMAD Forum (Presentation) (June 2017), p. 18, accessed on 2/2/2021, at: https://cutt.ly/jjR480O 
الهجرة القسرية والمجتمعات المضيفة(75). كما ينظم البرنامج لقاءات افتراضية لعرض نتائج استطلاعاته على شركائه وأصحاب المصلحة المعنيين باللجوء في المنطقة من أجل تشارك نتائجها.

\section{د. التخفيف من هواجس التوتر والنزاع المجتمعي}

تفاعلًا مع هواجس المدن المضيفة واللاجئين بخصوص احتمال نشوب توترات، واهتزاز الاستقرار الاجتماعي، ودعوتهم

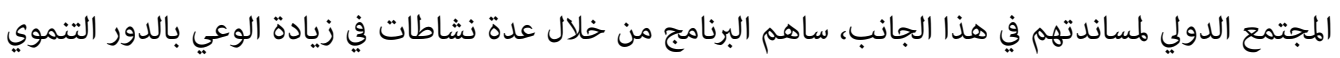

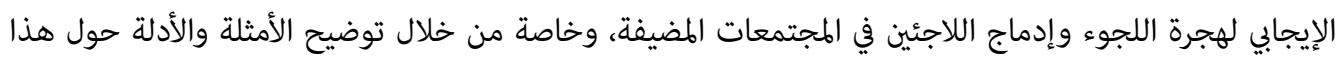
الموضوع ونشرها، بما يصحح المقاربات، ويخفف التوجسات. وقد نشر شركاء البرنامج مساهمة في إحدى الحوليات، توضح المزايا التي يجذبها المستثمرون السوريون للمدن المضيفة للاجئين من مقاربة محلية (76).

يبقى نشر مثل هذه المساهمات مهمًا، ولكنه غير كافٍ، إذ ينبغي أن يتم تحويل مضمونه إلى مادة إعلامية وتوعوية

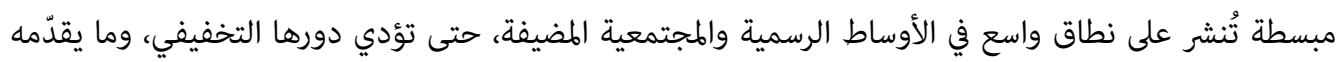
برنامج التعاون اللامركزي هنا هو مساهمات نوذجية ليستثمرها المعنيون.

\section{هـ. سد الحاجة إله التوجيه}

وفر البرنامج لبلديات الدول الخمس المشمولة أطرًا متعددة ومنسجمة للتشاور والتنسيق والتوجيه، بما يعوضها عن غياب السياسات الوطنية والمحلية المناسبة. فشبكة التعلم ومنصة التبادل الافتراضي، ومجموعات التوأمة واللجان

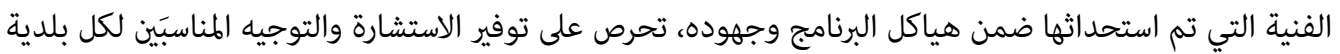
بحسب ظروفها واحتياجاتها وسجل الهجرة واللجوء الخاص بها.

كما أن المبادئ التي تعمل بها هذه البرامج ومشاريعها تتحول في الغالب إلى مبادئ مرشدة ومؤسسة طبادرات تعاون

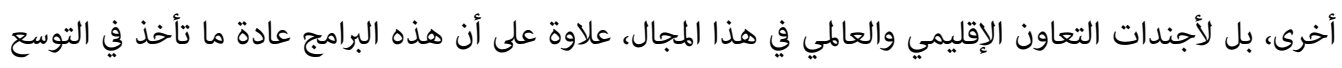

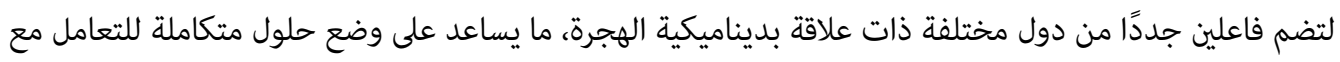
هذا المشكل، وهو ما عرفه أيضًا هذا البرنامج الآخذ في التوسع.

\section{و. سد الحاجة إله التنسيق بين الفاعلين وأصحاب المصلحة ومعهم}

يمثّل هذا البرنامج مظلة لتأطير عديد المبادرات والتدخلات والمشاريع، ويسمح بالتنسيق بين مختلف المتدخلين

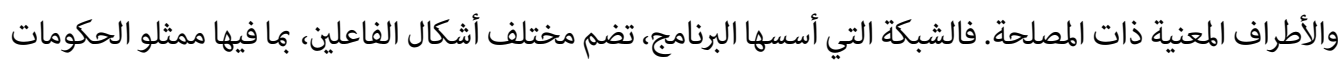
المركزية للدول المعنية وممثلو اللاجئين ومنظمات المجتمع المدني، وهي الفضاء الذي يلتقي فيه هؤلاء الفاعلون

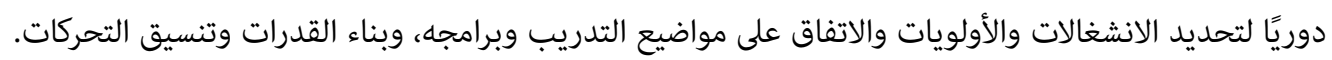

Bollag, p. 37 معرفة حصيلة هذه الأدوات، ينظر 75

76 John Speakman, Janette Uhlmann \& Gilda Borriello, "Mobilization of Syrian Investors and Private Sector to Boost Local Economic Development in Refugee Hosting Municipalities," in: Migration and Inclusive Rural Development in the Mediterranean (Paris: CIHEAM; Presses de Sciences Po; AFD, 2019), pp. 267-291. 


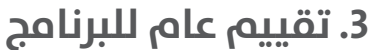

لا يسمح حجم هذا البرنامج وتعدد صور استجابته لأزمة اللاجئين وتكاملها، في هذا السياق، بتقديم تقييم مفصل

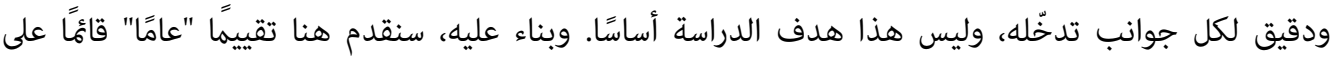

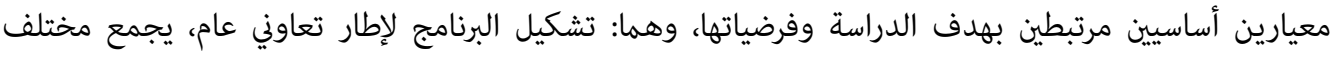

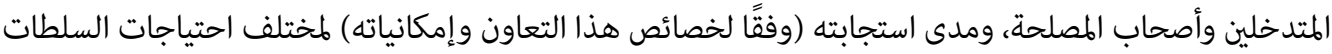
المحلية المضيفة في إطار تكفّلها بتقديم الخدمات الأساسية للمجتمع المحلي واللاجئين معًا. إنّ اعتماد برامج للتعاون اللامركزي في هذا المجال يعتبر، في حد ذاته، استجابة لعديد التطلعات والحاجات المعبّر

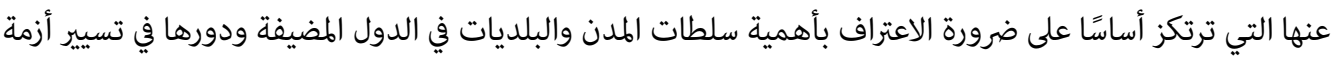
اللجوء، واستشارتها وإشراكها في جهود التكفل باللاجئين الحضريين المقيمين على أقاليمها، ودعم قدرتها على الصمود ومواجهة ضغط زيادة الطلب على الخدمات العامة المحلية الأساسية الذي يتسببون فيه. يضاف إلى ذلك طبيعة الجهة المشرفة على البرنامج، أي "مركز التكامل المتوسطي"، بوصفه هيئةً أو محفلاً متعدد

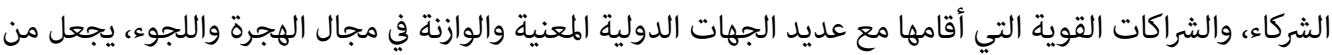
برامجه أطرًا متكاملة ومهمة جدًا لتأطير التعاون الأوروبي - العربي (المتوسطي) في عديد مجالات التهات اهتمامه، وخاصة اللجوء، وبناء رؤية مشتركة لمختلف المتدخلين فيها على مستوى الدول المضيفة.

ومن حيث توجهه ومقاربة عمله، تشير التقييمات الأولية إلى أن هذا البرنامج بتوجهه "الإدماجي"، يتماشى مع

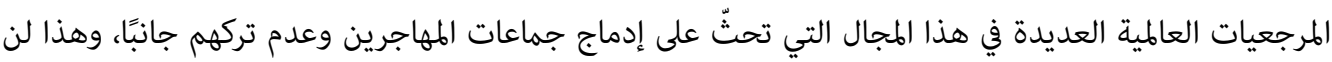
يكون ممكنًا من دون دعم قدرات السلطات المحلية للمدن المضيفة، ومرافقتها عبر برامج تعاون، تتم هندست إمستها

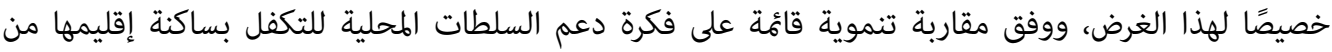

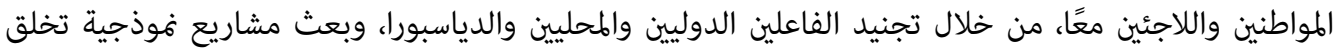

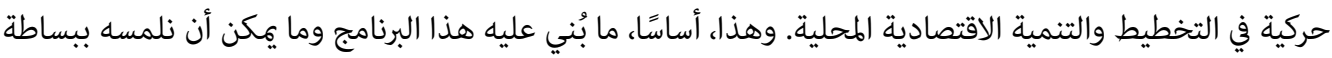
من خلال أهدافه وبرامجه الفرعية ونشاطاته العملية المنجزة حتى الآن.

وتوضح المقارنة البسيطة بين ما يُتوقع من مثل هذا النوع من التعاون واحتياجات البلديات المضيفة، وما قدَّمه هذا

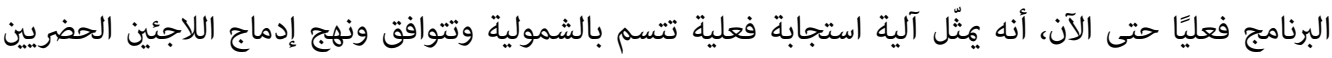

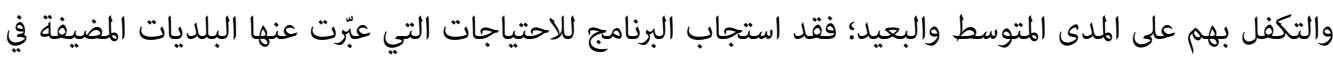

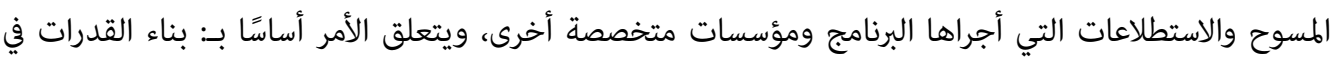
مجال التخطيط والتنفيذ للتنمية المحلية والمشاريع الإدماجية، وذلك عبر ندواته وورشاته التدريبية العديدة التي وتئي تحورت حول التخطيط للتنمية المحلية في سياق اللجوء وابتكار طرق لدعم الخدمات العامة، وجمع وتقديم البيانات

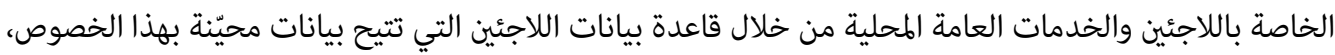
وتوفير فضاء للتعلم من الأقران الأوروبيين ومن المنطقة من خلال شبكة التعلم ومنصة التبادل الإلكتروني الخاصة بهاتيا

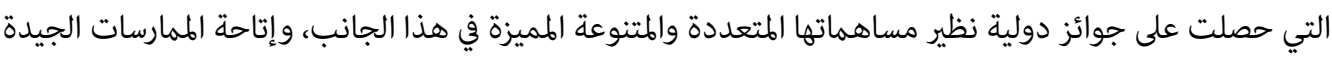
والتجارب بطريقة توطينها وتطبيقها، من خلال خلاصات التجارب المعدّة وفق منهجية مناسبة لهذا الغرض ومتاحة 
للعامة، ووضع أطرٍ لجلب الدعم والتمويل للسلطات المحلية والخدمات العامة، عبر مؤتمرات جمع التبرعات وتعبئة القطاع الخاص المحلي ورجال الأعمال التابعين للدياسبورا من أجل خلق فرص عمل ومشاريع إدماجية، والمساهمة في تغيير الصورة النمطية الخاطئة حول اللاجئين وتوضيح دورهم التنموي عبر دراسات رصينة في هذا الجانب يمكن إنماتِ استغلالها إعلاميًا لهذا الغرض، وتوفير التوجيه السياسي والسياساتي للسلطات المحلية المضيفة عبر اللقاءات التشاورية والتوجيه والتجارب وأدلة التعامل المختلفة التي يوفرها البرنامج وشركاؤه والطبادئ التي يقوم عليها. كما يُعزى صمود البلديات المضيفة وتكيفها نسبيًا مع الأزمة الحادة إلى تبادل المعارف والخبرات وتنسيق الاستجابات عبر عديد المبادرات، من بينها برنامج اللاجئين والمجتمعات المتوسطية المضيفة. ويُتوقع من البلديات المنخرطة في

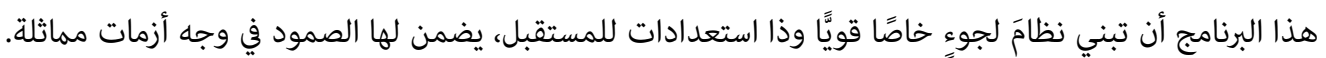
وحتى بالنسبة إلى مشكل التنسيق بين برامج التعاون الذي يبرز في مثل هذه الحالات، نتيجة تعدد المبادرات

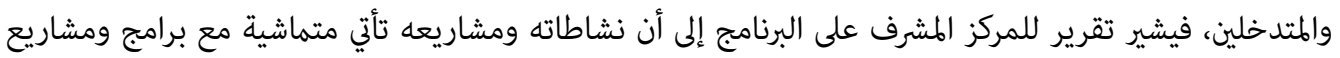
الفاعلين والشركاء الآخرين، ويقدم بعض الأمثلة الملموسة (77). كما يحضر المركز المشرف ويشارك في عديد النشاطات والأحداث التي ينظمها الشركاء في هذا المجال ومجالات ذات صلة؛ فخلال عام 2017 حضر خمسة أحداث دولية (78.

\section{خاتمة}

تَثّل برامج التعاون اللامركزي الأوروبي - العربي الأخرى "أطرًا تنفيذية" بالغة الأهمية، من حيث كونها تكمّل بقية الأطر القانونية العالمية، والسياسية الوطنية إن وُجدت، وتساهم في مخللتها، أو تعويض هذه الأخيرة حال غيابها،

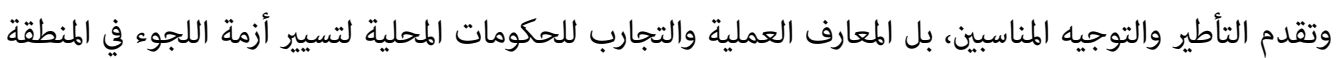
العربية، وبناء نظام للصمود مستقبلًا.

مع ذلك، من المهم جدًا هنا التأكيد أن استجابة هذا النمط من التعاون ومساهمته في شكله البرامجي، لا تلبيان

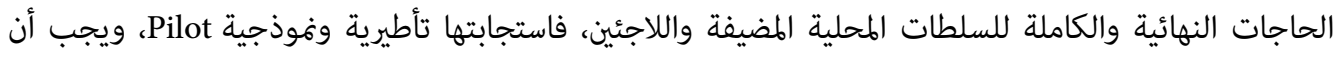
تُفهم وتُقيّم على هذا الأساس؛ أي إن وجود هذه البرامج يعني وجود أطر Frameworks متمحورة حول دور السلطات المحلية، يمكن أن يتخذها الفاعلون وأصحاب المصلحة أساسًا للتشاور وتنسيق تدخلاتهم وتشارك الخبرات والتجارب وصنع السياسات والتخطيط للتحركات. وهي أطر غير ملزمة وقائمة على أساس الرغبة والإرادة وتقدير

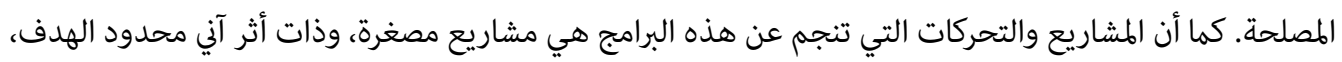
منها اختبار مخرجات التدريب وعملية بناء القدرات والتعرف إلى الصعوبات والمشكلات العملية التي قد تعترض تحركات السلطات المحلية في سياقات اللجوء، ومن ثم توسيع التحرك والأثر. ويبقى الرهان على إرادة الحكومات

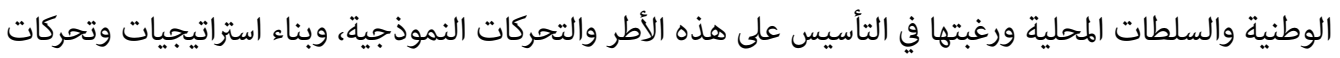

78 Center for Mediterranean Integration, CMI Annual Report 2017: Changing Realities, Strengthened Collaboration (Marseille: Center for Mediterranean Integration, 2017), p. 28. 
خاصة تبعًا لذلك، خاصة أن انخراطها في مثل هذه البرامج يجعلها مؤهلة للاستفادة من التمويل الدولي الذي تمنحه برامج التعاون الدولي ومشاريعه المختلفة، وهذا يُعتبر من الآثار والكاسب المستدامة لبادرات التعاون اللامركزي. تبقى هذه البرامج معدودة ومتواضعة، مقارنة بمبادرات المنظمات غير الحكومية التي تمتلك حرية حركة أكبر من

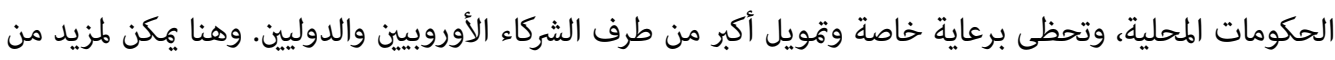

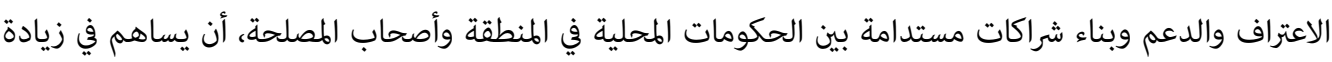
نطاق أطر التعاون اللامركزي في مجال الهجرة القسرية وغيره من المجاءلات. يجب أن يكون تكثيف هذه البرامج وتوسيعها وسيلة لبناء قدرات مدننا وصمودها أمام أزمات الهجرة وغيرها من فن الهات

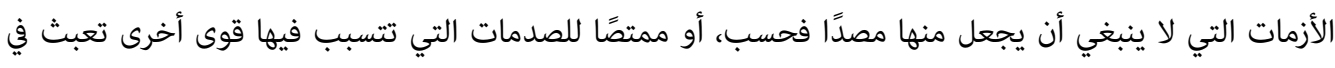
المنطقة وبها، ولا أن تساير أجندات تسعى لإعادة ترتيب بشري وسكاني للمنطقة على حساب الوضع القائم في بلداننا،

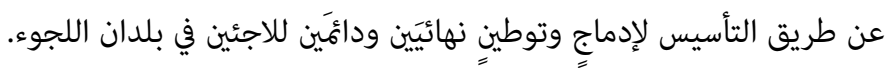




\section{العربية}

الأمم المتحدة، الجمعية العامة. الاتفاق العالمي من أجل الهجرة الآمنة والمنظمة والنظامية. 2018/11/19. الأمم المتحدة. الاتفاق العالمي بشأن اللاجئين (2018). في: https:/bit.ly/2OdAHNF

مباركية، منير. محاضرات في التعاون اللامركزي المتوسطي. مطبوعة بيداغوجية محكمة موجهة لطلبة الماجستير في برنامج دراسات متوسطية. عنابة: جامعة عنابة، معاضية 2016.

الأجنبية

Araya, Mariel \& Xavier Godàs. Migrations: A New Era of Co-peration between Local Governments. Montevideo: Observatorio de Cooperación Descentralizada UE-AL, 2008.

Bollag, Burton (ed.). CMI Annual Report 2018. Marseille: Center for Mediterranean Integration, 2018. Center for Mediterranean Integration. Best Practices in Hosting Refugees. Marseille: CMI, 2017. at: https://bit.ly/3ch97cj

. CMI Annual Report 2017: Changing Realities, Strengthened Collaboration. Marseille: Center for Mediterranean Integration, 2017.

. Experiences in Hosting Refugees: Local Economic Development in Host Communities.

Marseille: CMI, 2018. at: https://bit.ly/3cl5DFY

Ciacci, Silvia. "The Partnership with Local Authorities in Responding to Humanitarian Crisis: The case of Lebanon, Lessons learned and recommendations, Part Two." Oxfam Italia (September 2014). at: https://bit.ly/36SEQNC

"Cities of Refuge in the Middle East: Bringing an Urban Lens to the Forced Displacement Challenge." Policy Note. The World Bank. 14/9/2017. at: https://bit.ly/3jtekiU

Dick, Eva \& Jana Kuhnt. "The Neglected Role of Cities in the Global Compact on Refugees." The Current Column. German Development Institute (January 2019). at: https://bit.ly/3q6Es5S

De Losada Passols, Agustí Fernández. "Shaping a New Generation of Decentralised Cooperation for Enhanced Effectiveness and Accountability." PLATFORMA \& Conference of Peripheral Maritime Regions (CPMR) (October 2017). at: https://bit.ly/3jAyYh5

"Engaging Municipalities in the Response to the Syrian Refugee Crisis in Lebanon." Policy Brief. Mercy Corps - Lebanon (March 2014). at: https://bit.ly/3rwGnkf 
Grandi, Francesca, Kholoud Mansour \& Kerrie Holloway. Dignity and displaced Syrians in Lebanon: 'There is no karama here'. London: Humanitarian Policy Group; Overseas Development Institute, 2018.

International Organization for Migration. World Migration Report 2018. Geneva: IOM, 2017. Mahdi, Dima. "Understanding Refugee Politics in Lebanon and Calls for Repatriation." LCPS Policy Briefs. no. 30. The Lebanese Center for Policy Studies (October 2017). at: https://bit.ly/3tFHGPC "Mediterranean Refugees and Host Communities Program." Center for Mediterranean Integration. Amman Peer to Peer Learning Workshop (Presentation). 31/5/2016. at: https://bit.ly/3psRnhV Migration and Inclusive Rural Development in the Mediterranean. Paris: CIHEAM; Presses de Sciences Po; AFD, 2019.

Muggah, Robert \& Adriana Erthal Abdenur. "Refugees and the City: The Twenty-first-century Front Line." World Refugee Council Research Paper. no. 2. World Refugee Council \& Center for International Governance Innovation (July 2018). at: https://bit.ly/3p31oRU

"Social Affairs: Refugee Reception and Integration in Cities." Eurocities (March 2016). at: https://bit.ly/36PVk9m

Uhlmann, Janette. "Center for Mediterranean Integration: Perspectives from the Mediterranean Host Municipalities Network." KNOMAD Forum (Presentation). (June 2017). at: https://cutt.ly/jjR480O

United Nations High Commissioner for Refugees. Global Trends: Forced Displacement in 2018. Geneva: UNHCR, 2019.

"Urban Migration: Strengthening Cooperation with Civil Society." Peer Learning Note. no. 25. UCLG Learning (2019). at: http://bit.ly/2MASWMP

"Urban Refuge: How Cities Are Building Inclusive Communities." International Rescue Committee (2018). at: https://bit.ly/3oTcYPC

YearBook for Decentralised Cooperation: 2007. Montevideo: Observatorio de Cooperación Descentralizada UE-AL, 2008. 